\title{
TITLE:
}

\section{Cylindrical Couette flow of a vapor- gas mixture: Ghost effect and bifurcation in the continuum limit}

\author{
$\operatorname{AUTHOR}(S)$ :
}

Yoshida, Hiroaki; Aoki, Kazuo

\section{CITATION:}

Yoshida, Hiroaki ...[et al]. Cylindrical Couette flow of a vapor-gas mixture: Ghost effect and bifurcation in the continuum limit. PHYSICS OF FLUIDS 2006, 18(8): 087103.

\section{ISSUE DATE:}

2006-08

URL:

http://hdl.handle.net/2433/39787

\section{RIGHT:}

Copyright 2006 American Institute of Physics. This article may be downloaded for personal use only. Any other use requires prior permission of the author and the American Institute of Physics. 


\title{
Cylindrical Couette flow of a vapor-gas mixture: Ghost effect and bifurcation in the continuum limit
}

\author{
Hiroaki Yoshida \\ Department of Aeronautics and Astronautics and Advanced Research Institute of Fluid Science \\ and Engineering, Graduate School of Engineering, Kyoto University, Kyoto 606-8501, Japan \\ Kazuo Aoki \\ Department of Mechanical Engineering and Science and Advanced Research Institute of Fluid Science \\ and Engineering, Graduate School of Engineering, Kyoto University, Kyoto 606-8501, Japan
}

(Received 25 May 2006; accepted 13 July 2006; published online 23 August 2006)

\begin{abstract}
A binary mixture of gases is confined in a gap between two coaxial circular cylinders rotating at different angular velocities. One of the component gases is the vapor of the substance that forms the cylinders, so that evaporation or condensation (or sublimation) of the vapor may take place on the surfaces of the cylinders. The other component is a noncondensable gas that neither evaporates nor condenses on the surfaces. Axisymmetric and axially uniform flows (the cylindrical Couette flow) of such a mixture are investigated on the basis of kinetic theory with special interest in the continuum (or fluid-dynamic) limit in which the Knudsen number goes to zero. The fluid-dynamic system that describes the behavior of the mixture is derived by a formal but systematic asymptotic analysis of the Boltzmann system. The resulting system shows some nontrivial phenomena such as the ghost effect and the flow bifurcation. These phenomena are also demonstrated by a Monte Carlo simulation for small Knudsen numbers using the Boltzmann equation. (C) 2006 American Institute of Physics. [DOI: 10.1063/1.2336455]
\end{abstract}

\section{INTRODUCTION}

Axisymmetric and axially uniform flow between two rotating coaxial circular cylinders is a textbook example known as cylindrical Couette flow in classical fluid dynamics. It is also one of the basic flows for a rarefied gas and has been investigated on the basis of kinetic theory (see, for example, Refs. 1-11). The flow is a simple time-independent and spatially one-dimensional flow, and it exhibits neither instability nor bifurcation unless the axial uniformity is released. However, if the gas is a vapor of the substance that forms the cylinders and it undergoes evaporation or condensation (or sublimation) on the surfaces of the cylinders, the situation changes dramatically. The flow exhibits bifurcation for relatively small Knudsen numbers even under the constraint of axial uniformity. ${ }^{12-15}$ That is, there appear two or more different solutions for a certain range of parameters, such as the rotation speeds, the radii of the cylinders, and the Knudsen number. The structure of this bifurcation was investigated in detail by a formal but systematic asymptotic analysis of the Boltzmann equation with a delicate parameter setting in the case of slow rotation and small Knudsen numbers, and the essential features of the bifurcation were clarified. ${ }^{14}$ One of the cases considered in Ref. 14 was then investigated mathematically in Ref. 16, and a rigorous proof of the existence of the bifurcation in the asymptotic limit was given.

Now let us consider the continuum (or fluid-dynamic) limit where the Knudsen number goes to zero in the case with evaporation and condensation on the cylinders. When the inner cylinder is rotating and the outer cylinder is at rest, there appear three different types of solution in a wide parameter range. The first type exhibits evaporation on the in- ner cylinder and condensation on the outer, the second type exhibits condensation on the inner cylinder and evaporation on the outer, and the last type exhibits no evaporation or condensation on the cylinders. ${ }^{12}$ The first and second types are described by the Euler equations with appropriate boundary conditions for evaporation and condensation. ${ }^{17}$ For the third type, since there is no evaporation or condensation, it is natural to think that the flow field between the cylinders is the same as that of the ordinary cylindrical Couette flow. It is, however, not correct. The infinitesimal evaporation and condensation in this case have a significant effect on the flow field, such as the tangential velocity and temperature fields. ${ }^{18}$ This is an example of the ghost effect that was pointed out in Ref. 19 and investigated extensively in successive papers (see, e.g., Refs. 18 and 20-25). The fluid-dynamic system that describes the third type of solution was derived systematically from the Boltzmann equation in Ref. 18, and the deformation of the tangential component of the flow velocity caused by the ghost effect was clarified. Direct numerical analysis of the Boltzmann equation or its model equation for very small Knudsen numbers was able to give numerical solutions that correspond to the first and the second type mentioned above. ${ }^{12,13,15}$ However, it could not show the solution corresponding to the third type, ${ }^{12,13}$ most probably because of the instability of the solution. When the outer cylinder is rotating and the inner cylinder is at rest, there is no bifurcation of the flow in the continuum limit, and the solution without evaporation or condensation (i.e., the solution with the ghost effect) appears for the rotation speed higher than a critical value. The solution corresponding to this so- 
lution was obtained by the direct numerical analysis for small Knudsen numbers. ${ }^{12,18}$

In the present study, we consider the same problem, the cylindrical Couette flow of the vapor with evaporation and condensation on the surfaces of the cylinders. The difference from the previous study is that we consider the case where another noncondensable gas, which neither evaporates nor condenses on the surfaces of the cylinders, is also contained in the gap of the two cylinders. According to the study of the plane Couette flow of the mixture of a vapor and a noncondensable gas, ${ }^{26}$ we can expect, in the cylindrical Couette flow, that evaporation and condensation are blocked by the noncondensable gas and vanish in the continuum limit in any parameter range. Therefore, the solution that exhibits the ghost effect caused by the infinitesimal evaporation and condensation is the natural and only possible solution when there is noncondensable gas. We investigate this problem and derive the fluid-dynamic system that describes the behavior of the mixture in the continuum limit by a systematic asymptotic analysis of the Boltzmann system. As expected, the resulting fluid-dynamic system shows the ghost effect. More specifically, although evaporation and condensation of the vapor vanish in the continuum limit, it changes the flow field, such as the profile of the tangential component of the flow velocity, dramatically. In addition, such a type of solution exhibits bifurcation in a certain parameter range. The ghost effect as well as the bifurcation of solution is investigated in detail on the basis of the numerical solution of the fluid-dynamic system. Furthermore, these results are compared with the Monte Carlo simulation of the Boltzmann equation for small Knudsen numbers.

\section{FORMULATION OF THE PROBLEM}

\section{A. Problem}

Let us consider a binary mixture of a vapor ( $A$ component) and a noncondensable gas ( $B$ component) confined in the gap between two coaxial circular cylinders made of the condensed phase of the vapor and rotating at different angular velocities. The vapor may evaporate or condense on the surfaces of the cylinders, whereas the noncondensable gas undergoes ordinary reflection without evaporation or condensation there. The radius, temperature, and surface velocity of the inner cylinder are denoted by $L_{\mathrm{I}}, T_{\mathrm{I}}$, and $V_{\mathrm{I}}$, respectively, and the corresponding quantities of the outer cylinder by $L_{\mathrm{II}}$, $T_{\mathrm{II}}$, and $V_{\mathrm{II}}$.

Restricting ourselves to the case of axisymmetric and axially uniform flows, we investigate the steady behavior of the mixture on the basis of the Boltzmann equation with special interest in the continuum limit where the Knudsen number vanishes.

\section{B. Basic equations}

Let us introduce the cylindrical coordinate system $(r, \theta, z)$ with the $z$ axis along the common axis of the cylinders. Let $\xi$ be the molecular velocity and $\xi_{r}, \xi_{\theta}$, and $\xi_{z}$ its $r$, $\theta$, and $z$ components, $F^{\alpha}(r, \boldsymbol{\xi})$ the velocity distribution func- tion of the molecules of the $\alpha$ component $(\alpha=A, B)$. In what follows, we will use the indices $\alpha$ and $\beta$ to represent the components of the mixture, i.e., $\alpha, \beta=A, B$.

The Boltzmann equation ${ }^{27-29}$ in the present problem is written in the following form:

$$
\xi_{r} \frac{\partial F^{\alpha}}{\partial r}+\frac{\xi_{\theta}^{2}}{r} \frac{\partial F^{\alpha}}{\partial \xi_{r}}-\frac{\xi_{r} \xi_{\theta}}{r} \frac{\partial F^{\alpha}}{\partial \xi_{\theta}}=\sum_{\beta=A, B} J^{\beta \alpha}\left(F^{\beta}, F^{\alpha}\right),
$$

where $J^{\beta \alpha}$ is the collision integral defined by

$$
\begin{aligned}
J^{\beta \alpha}(F, G)= & \int\left[F\left(\boldsymbol{\xi}_{*}^{\prime}\right) G\left(\boldsymbol{\xi}^{\prime}\right)-F\left(\boldsymbol{\xi}_{*}\right) G(\boldsymbol{\xi})\right] \\
& \times B^{\beta \alpha}(|\mathbf{e} \cdot \boldsymbol{V}| / V, V) \mathrm{d} \Omega(\mathbf{e}) \mathrm{d} \boldsymbol{\xi}_{*},
\end{aligned}
$$

with

$$
\begin{aligned}
& \boldsymbol{\xi}^{\prime}=\boldsymbol{\xi}+\frac{\mu^{\beta \alpha}}{m^{\alpha}}(\mathbf{e} \cdot \boldsymbol{V}) \mathbf{e}, \quad \boldsymbol{\xi}_{*}^{\prime}=\boldsymbol{\xi}_{*}-\frac{\mu^{\beta \alpha}}{m^{\beta}}(\mathbf{e} \cdot \boldsymbol{V}) \mathbf{e}, \\
& \boldsymbol{V}=\boldsymbol{\xi}_{*}-\boldsymbol{\xi}, \quad V=|\boldsymbol{V}|, \quad \mu^{\beta \alpha}=\frac{2 m^{\alpha} m^{\beta}}{m^{\alpha}+m^{\beta}} .
\end{aligned}
$$

Here, $\boldsymbol{\xi}_{*}$ is the integration variable corresponding to $\boldsymbol{\xi}$, and $\mathrm{d} \boldsymbol{\xi}_{*}=\mathrm{d} \xi_{*_{r}} \mathrm{~d} \xi_{* \theta} \mathrm{d} \xi_{*_{z}} ; \mathbf{e}$ is the unit vector and $\mathrm{d} \Omega(\mathbf{e})$ is the solidangle element in its direction; $m^{\alpha}$ is the mass of a molecule of the $\alpha$ component; the domain of integration with respect to $\mathbf{e}$ is all directions, and that with respect to $\boldsymbol{\xi}_{*}$ is its whole space; $B^{\beta \alpha}(|\mathbf{e} \cdot \boldsymbol{V}| / V, V)$ is a non-negative function of $|\mathbf{e} \cdot \boldsymbol{V}| / V$ and $V$, whose functional form is determined by the intermolecular force.

In order to describe the boundary condition on the cylinders, we need to introduce the saturation number densities $n_{\mathrm{I}}$ and $n_{\mathrm{II}}$ of the vapor molecules at temperatures $T_{\mathrm{I}}$ and $T_{\mathrm{II}}$, respectively. For each substance, the saturation vapor pressure (or number density) is a function of the temperature only, determined by the Clausius-Clapeyron relation. ${ }^{30}$ Thus, $n_{\mathrm{I}}$ and $n_{\mathrm{II}}$ are determined by $T_{\mathrm{I}}$ and $T_{\mathrm{II}}$, respectively. However, we can regard $n_{\mathrm{I}}$ and $n_{\mathrm{II}}$ as parameters independent of $T_{\mathrm{I}}$ and $T_{\mathrm{II}}$ if we keep the freedom of choosing the substance of the vapor. For the purpose of describing the boundary conditions on the inner and outer cylinders in a unified fashion, we define the symbols $\sigma_{w}, n_{w}, V_{w}$, and $T_{w}$ by

$$
\sigma_{w}=1, \quad n_{w}=n_{\mathrm{I}}, \quad V_{w}=V_{\mathrm{I}}, \quad T_{w}=T_{\mathrm{I}},
$$

at $r=L_{\mathrm{I}}$, and

$$
\sigma_{w}=-1, \quad n_{w}=n_{\mathrm{II}}, \quad V_{w}=V_{\mathrm{II}}, \quad T_{w}=T_{\mathrm{II}},
$$

at $r=L_{\mathrm{II}}$. The boundary conditions at $r=L_{\mathrm{I}}$ and $L_{\mathrm{II}}$ are expressed in the following form: ${ }^{22,29,31}$

$$
F^{\alpha}(r, \boldsymbol{\xi})=g_{w}^{\alpha}(\boldsymbol{\xi})+\int_{\sigma_{w} \xi_{*}<0} K_{w}^{\alpha}\left(\boldsymbol{\xi}, \boldsymbol{\xi}_{*}\right) F^{\alpha}\left(r, \boldsymbol{\xi}_{*}\right) \mathrm{d} \boldsymbol{\xi} *
$$

$\left(\right.$ for $\left.\sigma_{w} \xi_{r}>0\right)$,

where the source term $g_{w}^{\alpha}(\boldsymbol{\xi})$ and the scattering kernel $K_{w}^{\alpha}\left(\boldsymbol{\xi}, \boldsymbol{\xi}_{*}\right)$ are given functions, the functional forms of which are determined by the nature of the boundary. The $g_{w}^{\alpha}$ and $K_{w}^{\alpha}$ need to satisfy the following conditions: 


$$
\begin{aligned}
& g_{w}^{A}(\boldsymbol{\xi}) \geqslant 0 \quad\left(\sigma_{w} \xi_{r}>0\right), \\
& g_{w}^{B}(\boldsymbol{\xi})=0 \quad\left(\sigma_{w} \xi_{r}>0\right), \\
& K_{w}^{\alpha}\left(\boldsymbol{\xi}, \boldsymbol{\xi}_{*}\right) \geqslant 0 \quad\left(\sigma_{w} \xi_{r}>0, \quad \sigma_{w} \xi_{*_{r}}<0\right), \\
& \int_{\sigma_{w} \xi_{r}>0}\left|\xi_{r} / \xi_{*_{r}}\right| K_{w}^{B}\left(\boldsymbol{\xi}, \xi_{*}\right) \mathrm{d} \boldsymbol{\xi}=1 \quad\left(\sigma_{w} \xi_{*_{r}}<0\right),
\end{aligned}
$$

where $\mathrm{d} \boldsymbol{\xi}=\mathrm{d} \xi_{r} \mathrm{~d} \xi_{\theta} \mathrm{d} \xi_{z}$. Equation (7d) indicates that there is no net particle flux of the $B$ component across the surface of the cylinders, that is, the $B$ component is noncondensable. In the present problem, $g_{w}^{A}$ and $K_{w}^{A}$ depend on $n_{w}, V_{w}, T_{w}$, and $m^{A}$, and $K_{w}^{B}$ depends on $V_{w}, T_{w}$, and $m^{B}$. We assume that Eq. (6) is satisfied by the wall-state equilibrium distributions, i.e.,

$$
F_{w}^{\alpha}(\boldsymbol{\xi})=g_{w}^{\alpha}(\boldsymbol{\xi})+\int_{\sigma_{w} \xi_{*}<0} K_{w}^{\alpha}\left(\boldsymbol{\xi}, \boldsymbol{\xi}_{*}\right) F_{w}^{\alpha}\left(\boldsymbol{\xi}_{*}\right) \mathrm{d} \boldsymbol{\xi}_{*},
$$

with

$$
\begin{aligned}
& F_{w}^{A}(\boldsymbol{\xi})=\frac{n_{w}}{\left(2 \pi k_{B} T_{w} / m^{A}\right)^{3 / 2}} \exp \left(-\frac{\xi_{r}^{2}+\left(\xi_{\theta}-V_{w}\right)^{2}+\xi_{z}^{2}}{2 k_{B} T_{w} / m^{A}}\right), \\
& F_{w}^{B}(\xi)=\frac{c}{\left(2 \pi k_{B} T_{w} / m^{B}\right)^{3 / 2}} \exp \left(-\frac{\xi_{r}^{2}+\left(\xi_{\theta}-V_{w}\right)^{2}+\xi_{z}^{2}}{2 k_{B} T_{w} / m^{B}}\right),
\end{aligned}
$$

where $c$ is an arbitrary constant, and $k_{B}$ is the Boltzmann constant. We also assume that Eqs. (9a) and (9b) are the only equilibrium distributions that satisfy Eq. (8).

Let $n^{\alpha}, \boldsymbol{v}^{\alpha}=\left(v_{r}^{\alpha}, v_{\theta}^{\alpha}, 0\right), p^{\alpha}$, and $T^{\alpha}$ be the molecular number density, the flow velocity, the pressure, and the temperature of the $\alpha$ component. These macroscopic quantities are expressed in terms of the velocity distribution functions as

$$
\begin{aligned}
& n^{\alpha}=\int F^{\alpha} \mathrm{d} \boldsymbol{\xi} \\
& v_{r}^{\alpha}=\frac{1}{n^{\alpha}} \int \xi_{r} F^{\alpha} \mathrm{d} \boldsymbol{\xi}, \quad v_{\theta}^{\alpha}=\frac{1}{n^{\alpha}} \int \xi_{\theta} F^{\alpha} \mathrm{d} \boldsymbol{\xi}, \\
& p^{\alpha}=k_{B} n^{\alpha} T^{\alpha}=\frac{1}{3} \int\left[\left(\xi_{r}-v_{r}^{\alpha}\right)^{2}+\left(\xi_{\theta}-v_{\theta}^{\alpha}\right)^{2}+\xi_{z}^{2}\right] m^{\alpha} F^{\alpha} \mathrm{d} \boldsymbol{\xi} .
\end{aligned}
$$

In Eqs. (10a)-(10c) and in what follows, the domain of integration with respect to $\boldsymbol{\xi}$ is its whole space unless the contrary is stated. The number density $n$, the mass density $\rho$, the flow velocity $\boldsymbol{v}=\left(v_{r}, v_{\theta}, 0\right)$, the pressure $p$, and the temperature $T$ of the total mixture are defined by

$$
\begin{aligned}
& n=\int\left(F^{A}+F^{B}\right) \mathrm{d} \boldsymbol{\xi}, \\
& \rho=\int\left(m^{A} F^{A}+m^{B} F^{B}\right) \mathrm{d} \boldsymbol{\xi},
\end{aligned}
$$

$$
\begin{aligned}
& v_{r}=\frac{1}{\rho} \int \xi_{r}\left(m^{A} F^{A}+m^{B} F^{B}\right) \mathrm{d} \boldsymbol{\xi}, \\
& \begin{aligned}
& v_{\theta}=\frac{1}{\rho} \int \xi_{\theta}\left(m^{A} F^{A}+m^{B} F^{B}\right) \mathrm{d} \boldsymbol{\xi}, \\
& p=k_{B} n T= \frac{1}{3} \int\left[\left(\xi_{r}-v_{r}\right)^{2}+\left(\xi_{\theta}-v_{\theta}\right)^{2}+\xi_{z}^{2}\right] \\
& \times\left(m^{A} F^{A}+m^{B} F^{B}\right) \mathrm{d} \boldsymbol{\xi} .
\end{aligned}
\end{aligned}
$$

Therefore, they are expressed in terms of the macroscopic quantities of each component as

$$
\begin{aligned}
& n=n^{A}+n^{B}, \\
& \rho=m^{A} n^{A}+m^{B} n^{B},
\end{aligned}
$$

$v_{r}=\left(m^{A} n^{A} v_{r}^{A}+m^{B} n^{B} v_{r}^{B}\right) / \rho$

$$
v_{\theta}=\left(m^{A} n^{A} v_{\theta}^{A}+m^{B} n^{B} v_{\theta}^{B}\right) / \rho
$$

$$
p=\sum_{\alpha=A, B}\left[p^{\alpha}+m^{\alpha} n^{\alpha}\left(v_{r}^{\alpha}-v_{r}\right)^{2} / 3+m^{\alpha} n^{\alpha}\left(v_{\theta}^{\alpha}-v_{\theta}\right)^{2} / 3\right] .
$$

It should be noted that $p^{\alpha}$ and $T^{\alpha}$ are sometimes defined in a different way in the literature, i.e., by Eq. (10c) with $v_{r}^{\alpha}$ and $v_{\theta}^{\alpha}$ replaced by $v_{r}$ and $v_{\theta}$ of Eqs. (11c) and (11d).

To complete the physical setting of the problem, we need to specify a quantity associated with the amount of the noncondensable gas. As this parameter, we choose the average number density of the noncondensable gas:

$$
n_{\mathrm{av}}^{B}=\frac{1}{\pi\left(L_{\mathrm{II}}^{2}-L_{\mathrm{I}}^{2}\right)} \int_{-\pi}^{\pi} \int_{L_{\mathrm{I}}}^{L_{\mathrm{II}}} \int r F^{B} \mathrm{~d} \boldsymbol{\xi} \mathrm{d} r \mathrm{~d} \theta .
$$

By integrating both sides of Eq. (1) with respect to $\boldsymbol{\xi}$ over its whole space, we obtain

$$
r n^{\alpha} v_{r}^{\alpha}=\text { const },
$$

which expresses the mass conservation for each component gas. On the other hand, if we integrate Eq. (6) (with $\alpha=B$ ) multiplied by $\xi_{r}$ with respect to $\boldsymbol{\xi}$ over the space $\sigma_{w} \xi_{r}>0$ and take Eq. (7d) into consideration, we obtain $n^{B} v_{r}^{B}=0$ at $r=L_{\mathrm{I}}$ and $L_{\mathrm{II}}$. Thus we have

$$
n^{B} v_{r}^{B}=0 \quad\left(L_{\mathrm{I}} \leqslant r \leqslant L_{\mathrm{II}}\right) .
$$

\section{Dimensionless expressions}

To derive the dimensionless expressions of the basic system, we introduce the following dimensionless variables: 


$$
\begin{aligned}
& \hat{r}=\frac{r}{L_{\mathrm{I}}}, \quad \zeta=\left(\zeta_{r}, \zeta_{\theta}, \zeta_{z}\right)=\frac{\boldsymbol{\xi}}{V_{\mathrm{th}}}, \\
& \hat{F}^{\alpha}=\frac{V_{\mathrm{th}}^{3}}{n_{\mathrm{I}}} F^{\alpha}, \quad \hat{m}^{\alpha}=\frac{m^{\alpha}}{m^{A}}, \\
& \hat{n}^{\alpha}=\frac{n^{\alpha}}{n_{\mathrm{I}}}, \quad \hat{\boldsymbol{v}}^{\alpha}=\left(\hat{v}_{r}^{\alpha}, \hat{v}_{\theta}^{\alpha}, 0\right)=\frac{\boldsymbol{v}^{\alpha}}{V_{\mathrm{th}}}, \\
& \hat{p}^{\alpha}=\frac{p^{\alpha}}{p_{\mathrm{I}}}, \quad \hat{T}^{\alpha}=\frac{T^{\alpha}}{T_{\mathrm{I}}}, \\
& \hat{n}=\frac{n}{n_{\mathrm{I}}}, \quad \hat{\rho}=\frac{\rho}{\rho_{\mathrm{I}}}, \quad \hat{\boldsymbol{v}}=\left(\hat{v}_{r}, \hat{v}_{\theta}, 0\right)=\frac{\boldsymbol{v}}{V_{\mathrm{th}}}, \\
& \hat{p}=\frac{p}{p_{\mathrm{I}}}, \quad \hat{T}=\frac{T}{T_{\mathrm{I}}},
\end{aligned}
$$

where $V_{\text {th }}=\left(2 k_{B} T_{\mathrm{I}} / m^{A}\right)^{1 / 2}$ is the most probable speed of the molecules of the $A$ component in the equilibrium state at rest at temperature $T_{\mathrm{I}} ; \rho_{\mathrm{I}}=m^{A} n_{\mathrm{I}}$ and $p_{\mathrm{I}}=k_{B} n_{\mathrm{I}} T_{\mathrm{I}}$ are, respectively, the density and the pressure of the vapor in the saturated equilibrium state at rest at temperature $T_{\mathrm{I}}$.

Then the Boltzmann equation (1) becomes

$$
\begin{aligned}
& \mathcal{D} \hat{F}^{\alpha}=\frac{2}{\sqrt{\pi} \operatorname{Kn}_{\beta=A, B}} \sum^{\beta \alpha} \hat{J}^{\beta \alpha}\left(\hat{F}^{\beta}, \hat{F}^{\alpha}\right), \\
& \mathcal{D}=\zeta_{r} \frac{\partial}{\partial \hat{r}}+\frac{\zeta_{\theta}^{2}}{\hat{r}} \frac{\partial}{\partial \zeta_{r}}-\frac{\zeta_{r} \zeta_{\theta}}{\hat{r}} \frac{\partial}{\partial \zeta_{\theta}}, \\
& \hat{J}^{\beta \alpha}(f, g)=\int\left[f\left(\boldsymbol{\zeta}_{*}^{\prime}\right) g\left(\boldsymbol{\zeta}^{\prime}\right)-f\left(\boldsymbol{\zeta}_{*}\right) g(\boldsymbol{\zeta})\right] \\
& \quad \times \hat{B}^{\beta \alpha}(|\mathbf{e} \cdot \hat{\boldsymbol{V}}| / \hat{V}, \hat{V}) \mathrm{d} \Omega(\mathbf{e}) \mathrm{d} \boldsymbol{\zeta}_{*}, \\
& \boldsymbol{\zeta}^{\prime}=\boldsymbol{\zeta}+\frac{\hat{\mu}^{\beta \alpha}}{\hat{m}^{\alpha}}(\mathbf{e} \cdot \hat{\boldsymbol{V}}) \mathbf{e}, \quad \boldsymbol{\zeta}_{*}^{\prime}=\boldsymbol{\zeta}_{*}-\frac{\hat{\mu}^{\beta \alpha}}{\hat{m}^{\beta}}(\mathbf{e} \cdot \hat{\boldsymbol{V}}) \mathbf{e}, \\
& \hat{\boldsymbol{V}}=\boldsymbol{\zeta}_{*}-\boldsymbol{\zeta}, \quad \hat{V}=|\hat{\boldsymbol{V}}|, \quad \hat{\mu}^{\beta \alpha}=\frac{2 \hat{m}^{\alpha} \hat{m}^{\beta}}{\hat{m}^{\alpha}+\hat{m}^{\beta}}, \\
& \hat{B}^{\beta \alpha}(|\mathbf{e} \cdot \hat{\boldsymbol{V}}| / \hat{V}, \hat{V})=\frac{B^{\beta \alpha}}{B_{c}^{\beta \alpha}}, \quad K^{\beta \alpha}=\frac{B_{c}^{\beta \alpha}}{B_{c}^{A A}},
\end{aligned}
$$$$
B_{c}^{\beta \alpha}=\frac{1}{n_{\mathrm{I}}^{2}} \int F_{c}^{\alpha}(\boldsymbol{\xi}) F_{c}^{\beta}\left(\boldsymbol{\xi}_{*}\right) B^{\beta \alpha}(|\mathbf{e} \cdot \boldsymbol{V}| / V, V) \mathrm{d} \Omega(\mathbf{e}) \mathrm{d} \boldsymbol{\xi} \mathrm{d} \boldsymbol{\xi}_{*},
$$

$$
F_{c}^{\alpha}(\boldsymbol{\xi})=\frac{n_{\mathrm{I}}}{\left(2 \pi k_{B} T_{\mathrm{I}} / m^{\alpha}\right)^{3 / 2}} \exp \left(-\frac{\xi_{r}^{2}+\xi_{\theta}^{2}+\xi_{z}^{2}}{2 k_{B} T_{\mathrm{I}} / m^{\alpha}}\right) .
$$

Here, $\boldsymbol{\zeta}_{*}$ is the integration variable corresponding to $\zeta$, and $\mathrm{d} \zeta_{*}=\mathrm{d} \zeta_{{ }_{r}} \mathrm{~d} \zeta_{*} \mathrm{~d} \zeta_{* z}$; the domain of integration is the whole space of $\zeta_{*}$ and all directions of $\mathbf{e}[\mathbf{e}$ and $\mathrm{d} \Omega(\mathbf{e})$ are the same as in Eq. (2)]; Kn on the right-hand side of Eq. (17) is the characteristic Knudsen number defined by $\mathrm{Kn}=\ell_{c} / L_{\mathrm{I}}$, where $\ell_{c}=2 V_{\mathrm{th}} / \sqrt{\pi} n_{\mathrm{I}} B_{c}^{A A}$ is the characteristic mean free path, i.e., the mean free path of the molecules of the vapor in the equilibrium state at rest with temperature $T_{\mathrm{I}}$ and molecular number density $n_{\mathrm{I}}$ in the absence of the noncondensable gas.

When the intermolecular potential extends to infinity, the so-called angular cutoff is introduced in Eq. (23), that is, $B^{\beta \alpha}$ is set to be zero for the domain $|\mathbf{e} \cdot \mathbf{V}| / V \leqslant \delta(\delta$ is a specified constant) that corresponds to grazing collisions. It should be noted that the function $\hat{B}^{\beta \alpha}$ in general depends on the dimensionless parameter $U_{c}^{\beta \alpha} / k_{B} T_{\mathrm{I}}$, where $U_{c}^{\beta \alpha}$ is the characteristic size of the intermolecular potential for the interaction of a molecule of the $\alpha$ component with a molecule of the $\beta$ component. $^{22}$ This parameter is not shown explicitly in the above equations. When both component gases consist of hard-sphere molecules, $\hat{B}^{\beta \alpha}$ does not depend on this parameter, and $\hat{B}^{\beta \alpha}, K^{\beta \alpha}$, and $\ell_{c}$ are given by

$$
\begin{aligned}
\hat{B}^{\beta \alpha} & =\frac{\left(\hat{\mu}^{\beta \alpha}\right)^{1 / 2}}{4 \sqrt{2 \pi}}|\mathbf{e} \cdot \hat{\boldsymbol{V}}|, \\
K^{\beta \alpha} & =\frac{1}{\left(\hat{\mu}^{\beta \alpha}\right)^{1 / 2}}\left(\frac{d^{\beta}+d^{\alpha}}{2 d^{A}}\right)^{2},
\end{aligned}
$$

$$
\ell_{c}=\frac{1}{\sqrt{2} \pi\left(d^{A}\right)^{2} n_{\mathrm{I}}},
$$

where $d^{\alpha}$ is the diameter of a molecule of the $\alpha$ component.

Corresponding to Eqs. (4) and (5), we introduce the following symbols: at $\hat{r}=1$,

$$
\sigma_{w}=1, \quad \hat{n}_{w}=1, \quad \hat{V}_{w}=\frac{V_{\mathrm{I}}}{V_{\mathrm{th}}}, \quad \hat{T}_{w}=1,
$$

and at $\hat{r}=L_{\mathrm{II}} / L_{\mathrm{I}}$,

$$
\sigma_{w}=-1, \quad \hat{n}_{w}=\frac{n_{\mathrm{II}}}{n_{\mathrm{I}}}, \quad \hat{V}_{w}=\frac{V_{\mathrm{II}}}{V_{\mathrm{th}}}, \quad \hat{T}_{w}=\frac{T_{\mathrm{II}}}{T_{\mathrm{I}}} .
$$

The dimensionless form of the boundary conditions is as follows: at $\hat{r}=1$ and $L_{\mathrm{II}} / L_{\mathrm{I}}$,

$$
\hat{F}^{\alpha}(\hat{r}, \boldsymbol{\zeta})=\hat{g}_{w}^{\alpha}(\boldsymbol{\zeta})+\int_{\sigma_{w} \zeta_{*}<0} \hat{K}_{w}^{\alpha}\left(\boldsymbol{\zeta}, \boldsymbol{\zeta}_{*}\right) \hat{F}^{\alpha}\left(\hat{r}, \boldsymbol{\zeta}_{*}\right) \mathrm{d} \boldsymbol{\zeta}_{*}
$$

$\left(\right.$ for $\left.\sigma_{w} \zeta_{r}>0\right)$

where

$$
\hat{g}_{w}^{\alpha}=\frac{V_{\mathrm{th}}^{3}}{n_{\mathrm{I}}} g_{w}^{\alpha}, \quad \hat{K}_{w}^{\alpha}=V_{\mathrm{th}}^{3} K_{w}^{\alpha},
$$

and $\hat{g}_{w}^{\alpha}(\boldsymbol{\zeta})$ and $\hat{K}_{w}^{\alpha}\left(\boldsymbol{\zeta}, \boldsymbol{\zeta}_{*}\right)$ satisfy

$$
\begin{aligned}
& \hat{g}_{w}^{A}(\zeta) \geqslant 0 \quad\left(\sigma_{w} \zeta_{r}>0\right), \\
& \hat{g}_{w}^{B}(\zeta)=0 \quad\left(\sigma_{w} \zeta_{r}>0\right), \\
& \hat{K}_{w}^{\alpha}\left(\zeta, \zeta_{*}\right) \geqslant 0 \quad\left(\sigma_{w} \zeta_{r}>0, \sigma_{w} \zeta_{* r}<0\right),
\end{aligned}
$$




$$
\int_{\sigma_{w} \zeta_{r}>0}\left|\zeta_{r}\right| \zeta_{*_{r}} \mid \hat{K}_{w}^{B}\left(\zeta, \zeta_{*}\right) \mathrm{d} \zeta=1 \quad\left(\sigma_{w} \zeta_{*_{r}}<0\right)
$$

where $\mathrm{d} \zeta=\mathrm{d} \zeta_{r} \mathrm{~d} \zeta_{\theta} \mathrm{d} \zeta_{z}$. In addition, corresponding to Eqs. (8), (9a), and (9b), we have

$$
\begin{aligned}
& \hat{F}_{w}^{\alpha}(\boldsymbol{\zeta})=\hat{g}_{w}^{\alpha}(\boldsymbol{\zeta})+\int_{\sigma_{w} \xi_{*}<0} \hat{K}_{w}^{\alpha}\left(\zeta, \zeta_{*}\right) \hat{F}_{w}^{\alpha}\left(\boldsymbol{\zeta}_{*}\right) \mathrm{d} \zeta_{*}, \\
& \hat{F}_{w}^{A}(\boldsymbol{\zeta})=\frac{\hat{n}_{w}}{\left(\pi \hat{T}_{w}\right)^{3 / 2}} \exp \left(-\frac{\zeta_{r}^{2}+\left(\zeta_{\theta}-\hat{V}_{w}\right)^{2}+\zeta_{z}^{2}}{\hat{T}_{w}}\right), \\
& \hat{F}_{w}^{B}(\boldsymbol{\zeta})=\frac{\hat{c}}{\left(\pi \hat{T}_{w} / \hat{m}^{B}\right)^{3 / 2}} \exp \left(-\frac{\zeta_{r}^{2}+\left(\zeta_{\theta}-\hat{V}_{w}\right)^{2}+\zeta_{z}^{2}}{\hat{T}_{w} / \hat{m}^{B}}\right),
\end{aligned}
$$

where $\hat{c}$ is an arbitrary (dimensionless) constant.

It is seen from Eqs. (28), (29), (33), (34a), and (34b) that the parameters $n_{\mathrm{II}} / n_{\mathrm{I}}, V_{\mathrm{I}} / V_{\mathrm{th}}, V_{\mathrm{II}} / V_{\mathrm{th}}, T_{\mathrm{II}} / T_{\mathrm{I}}$, and $m^{B} / m^{A}$ are contained in the boundary condition. In general, however, $\hat{g}_{w}^{A}$ and $\hat{K}_{w}^{A}$ also depend on the reference temperature $T_{\text {I }}$, the reference number density $n_{\mathrm{I}}$, and the reference molecular mass $m^{A}$, and $\hat{K}_{w}^{B}$ also depends on $T_{\mathrm{I}}$ and $m^{A}$. In the case of the so-called complete condensation condition (see Refs. 22 and 29) for the vapor, and the diffuse reflection condition (see Refs. 22 and 29) for the noncondensable gas, $\hat{g}_{w}^{\alpha}$ and $\hat{K}_{w}^{\alpha}$ are given by

$$
\begin{aligned}
& \hat{g}_{w}^{A}(\zeta)=\frac{\hat{n}_{w}}{\left(\pi \hat{T}_{w}\right)^{3 / 2}} \exp \left(-\frac{\zeta_{r}^{2}+\left(\zeta_{\theta}-\hat{V}_{w}\right)^{2}+\zeta_{z}^{2}}{\hat{T}_{w}}\right), \\
& \hat{g}_{w}^{B}(\boldsymbol{\zeta})=0, \\
& \hat{K}_{w}^{A}\left(\boldsymbol{\zeta}, \zeta_{*}\right)=0, \\
& \hat{K}_{w}^{B}\left(\boldsymbol{\zeta}, \zeta_{*}\right)=-\frac{2}{\pi}\left(\frac{\hat{m}^{B}}{\hat{T}_{w}}\right)^{2} \sigma_{w} \zeta_{* r} \\
& \quad \times \exp \left(-\frac{\zeta_{r}^{2}+\left(\zeta_{\theta}-\hat{V}_{w}\right)^{2}+\zeta_{z}^{2}}{\hat{T}_{w} / \hat{m}^{B}}\right) .
\end{aligned}
$$

In this case, $\hat{g}_{w}^{\alpha}$ and $\hat{K}_{w}^{\alpha}$ depend only on $n_{\mathrm{II}} / n_{\mathrm{I}}, V_{\mathrm{I}} / V_{\mathrm{th}}, V_{\mathrm{II}} / V_{\mathrm{th}}$, $T_{\mathrm{II}} / T_{\mathrm{I}}$, and $\mathrm{m}^{B} / \mathrm{m}^{A}$ and do not depend on $T_{\mathrm{I}}, n_{\mathrm{I}}$, or $m^{A}$. The boundary condition on an evaporating or condensing surface has been investigated by different approaches, and some new models have been proposed rather recently. ${ }^{32-36}$

The dimensionless version of the relations between the macroscopic variables and the velocity distribution functions [Eqs. (10)-(12)] is given by

$$
\begin{aligned}
& \hat{n}^{\alpha}=\int \hat{F}^{\alpha} \mathrm{d} \zeta, \\
& \hat{v}_{r}^{\alpha}=\frac{1}{\hat{n}^{\alpha}} \int \zeta_{r} \hat{F}^{\alpha} \mathrm{d} \zeta, \quad \hat{v}_{\theta}^{\alpha}=\frac{1}{\hat{n}^{\alpha}} \int \zeta_{\theta} \hat{F}^{\alpha} \mathrm{d} \zeta,
\end{aligned}
$$

$$
\hat{p}^{\alpha}=\hat{n}^{\alpha} \hat{T}^{\alpha}=\frac{2}{3} \int\left[\left(\zeta_{r}-\hat{v}_{r}^{\alpha}\right)^{2}+\left(\zeta_{\theta}-\hat{v}_{\theta}^{\alpha}\right)^{2}+\zeta_{z}^{2}\right] \hat{m}^{\alpha} \hat{F}^{\alpha} \mathrm{d} \zeta,
$$

$$
\begin{aligned}
& \hat{n}=\int\left(\hat{F}^{A}+\hat{F}^{B}\right) \mathrm{d} \zeta=\hat{n}^{A}+\hat{n}^{B}, \\
& \hat{\rho}=\int\left(\hat{m}^{A} \hat{F}^{A}+\hat{m}^{B} \hat{F}^{B}\right) \mathrm{d} \zeta=\hat{m}^{A} \hat{n}^{A}+\hat{m}^{B} \hat{n}^{B}, \\
& \hat{v}_{r}=\frac{1}{\hat{\rho}} \int \zeta_{r}\left(\hat{m}^{A} \hat{F}^{A}+\hat{m}^{B} \hat{F}^{B}\right) \mathrm{d} \zeta=\frac{1}{\hat{\rho}}\left(\hat{m}^{A} \hat{n}^{A} \hat{v}_{r}^{A}+\hat{m}^{B} \hat{n}^{B} \hat{v}_{r}^{B}\right),
\end{aligned}
$$

$$
\hat{v}_{\theta}=\frac{1}{\hat{\rho}} \int \zeta_{\theta}\left(\hat{m}^{A} \hat{F}^{A}+\hat{m}^{B} \hat{F}^{B}\right) \mathrm{d} \zeta=\frac{1}{\hat{\rho}}\left(\hat{m}^{A} \hat{n}^{A} \hat{v}_{\theta}^{A}+\hat{m}^{B} \hat{n}^{B} \hat{v}_{\theta}^{B}\right),
$$

$$
\begin{aligned}
\hat{p}=\hat{n} \hat{T}= & \frac{2}{3} \int\left[\left(\zeta_{r}-\hat{v}_{r}\right)^{2}+\left(\zeta_{\theta}-\hat{v}_{\theta}\right)^{2}+\zeta_{z}^{2}\right] \\
& \times\left(\hat{m}^{A} \hat{F}^{A}+\hat{m}^{B} \hat{F}^{B}\right) \mathrm{d} \zeta \\
= & \sum_{\alpha=A, B}\left[\hat{p}^{\alpha}+\frac{2}{3} \hat{m}^{\alpha} \hat{n}^{\alpha}\left(\hat{v}_{r}^{\alpha}-\hat{v}_{r}\right)^{2}\right. \\
& \left.+\frac{2}{3} \hat{m}^{\alpha} \hat{n}^{\alpha}\left(\hat{v}_{\theta}^{\alpha}-\hat{v}_{\theta}\right)^{2}\right] .
\end{aligned}
$$

Here and in what follows, the domain of integration with respect to $\zeta$ is its whole space unless the contrary is stated.

The dimensionless forms of Eqs. (13)-(15) are, respectively,

$$
\begin{aligned}
& \frac{n_{\mathrm{av}}^{B}}{n_{\mathrm{I}}}=\frac{2}{\left(L_{\mathrm{II}} / L_{\mathrm{I}}\right)^{2}-1} \int_{1}^{L_{\mathrm{II}} L_{\mathrm{I}}} \hat{r} \hat{n}^{B} \mathrm{~d} \hat{r}, \\
& \hat{r} \hat{n}^{A} \hat{v}_{r}^{A}=\mathrm{const} \quad \text { and } \quad \hat{n}^{B} \hat{v}_{r}^{B}=0, \quad \text { for } 1 \leqslant \hat{r} \leqslant L_{\mathrm{II}} / L_{\mathrm{I}} .
\end{aligned}
$$

\section{ASYMPTOTIC ANALYSIS FOR SMALL KNUDSEN NUMBERS}

In this section, we consider the case where the Knudsen number $\mathrm{Kn}$ is small and carry out a systematic asymptotic analysis of the boundary-value problem, Eqs. (17) and (30), following the asymptotic theory developed by Sone (see, e.g., Refs. 18, 19, 22, and 37-39) as a guideline. The analysis here can be carried out in a way parallel to Ref. 26. For convenience, we use the following small parameter $\epsilon$ rather than $\mathrm{Kn}$ in this section:

$$
\epsilon=(\sqrt{\pi} / 2) \mathrm{Kn} \ll 1 .
$$




\section{A. Fluid-dynamic equations and boundary conditions}

First, we leave aside the boundary condition (30) and look for a solution in the form of a power series of $\epsilon$,

$$
\hat{F}^{\alpha}=\hat{F}_{0}^{\alpha}+\hat{F}_{1}^{\alpha} \epsilon+\cdots,
$$

assuming that it is moderately varying, i.e., it satisfies $\partial \hat{F}^{\alpha} / \partial \hat{r}=O\left(\hat{F}^{\alpha}\right)$. This solution (or expansion) is called the Hilbert solution (or Hilbert expansion). Substitution of Eq. (43) into Eqs. (39a)-(39h) gives the corresponding power series expansions of the macroscopic variables of each component and of the total mixture:

$$
\begin{aligned}
& h^{\alpha}=h_{0}^{\alpha}+h_{1}^{\alpha} \epsilon+\cdots, \\
& h=h_{0}+h_{1} \epsilon+\cdots,
\end{aligned}
$$

where the letter $h$ stands for $\hat{n}, \hat{\boldsymbol{v}}, \hat{T}$, etc. The coefficient functions $h_{m}^{\alpha}$ and $h_{m}$ are expressed in terms of $\hat{F}_{n}^{\alpha}(n \leqslant m)$. Their explicit forms for $m=0$ and 1 are given in Appendix A. After substituting Eq. (43) into Eq. (17) and arranging the power of $\epsilon$, we obtain a series of integral equations for the coefficients $\hat{F}_{m}^{\alpha}$ :

$$
\begin{aligned}
& \sum_{\beta=A, B} K^{\beta \alpha} \hat{J}^{\beta \alpha}\left(\hat{F}_{0}^{\beta}, \hat{F}_{0}^{\alpha}\right)=0 \\
& \sum_{\beta=A, B} K^{\beta \alpha}\left[\hat{J}^{\beta \alpha}\left(\hat{F}_{0}^{\beta}, \hat{F}_{m}^{\alpha}\right)+\hat{J}^{\beta \alpha}\left(\hat{F}_{m}^{\beta}, \hat{F}_{0}^{\alpha}\right)\right] \\
& \quad=\mathcal{D} \hat{F}_{m-1}^{\alpha}-\sum_{l=1}^{m-1} \sum_{\beta=A, B} K^{\beta \alpha} \hat{J}^{\beta \alpha}\left(\hat{F}_{l}^{\beta}, \hat{F}_{m-l}^{\alpha}\right)
\end{aligned}
$$

$$
(m=1,2, \ldots),
$$

where $\sum_{l=1}^{m-1}$ is set to be zero when $m=1$.

As is well known, ${ }^{28}$ the solution of Eq. (46) is a local Maxwellian distribution for each component with a common temperature and a common flow velocity. The solution is expressed in the following form using the leading order terms $\hat{n}_{0}^{\alpha}, \hat{v}_{r 0}, \hat{v}_{\theta 0}$, and $\hat{T}_{0}$ of the Hilbert expansion of the macroscopic quantities:

$$
\hat{F}_{0}^{\alpha}=\frac{\hat{n}_{0}^{\alpha}}{\left(\pi \hat{T}_{0} / \hat{m}^{\alpha}\right)^{3 / 2}} \exp \left(-\frac{\left(\zeta_{r}-\hat{v}_{r 0}\right)^{2}+\left(\zeta_{\theta}-\hat{v}_{\theta 0}\right)^{2}+\zeta_{z}^{2}}{\hat{T}_{0} / \hat{m}^{\alpha}}\right) .
$$

This gives the obvious relations

$$
\begin{aligned}
& \hat{v}_{r 0}^{A}=\hat{v}_{r 0}^{B}=\hat{v}_{r 0}, \quad \hat{v}_{\theta 0}^{A}=\hat{v}_{\theta 0}^{B}=\hat{v}_{\theta 0}, \\
& \hat{T}_{0}^{A}=\hat{T}_{0}^{B}=\hat{T}_{0} .
\end{aligned}
$$

On the other hand, from Eq. (41) we have

$$
\hat{n}_{0}^{B} \hat{v}_{r 0}^{B}=0 \quad\left(1 \leqslant \hat{r} \leqslant L_{\mathrm{II}} / L_{\mathrm{I}}\right) .
$$

The case where $\hat{n}_{0}^{B} \equiv 0$, i.e., there is no noncondensable gas in the leading order (of the Hilbert solution), has been investigated for general geometry in Ref. 40. In this case, a small amount of the noncondensable gas is blown away by the vapor flow and pushed against the surface where condensation of the vapor is taking place. As a result, the noncondensable component of a very small amount accumulates in a very thin layer adjacent to the condensing surface and may have a significant effect on the global vapor flow. ${ }^{40-42}$ In the present study, we consider the opposite case, i.e., the case where $\hat{n}_{0}^{B}>0$ in $1 \leqslant \hat{r} \leqslant L_{\mathrm{II}} / L_{\mathrm{I}}$ (the case where $\hat{n}_{0}^{B}$ vanishes in a certain interval of $\hat{r}$ can be handled in the framework of the present analysis). Then, $\hat{v}_{r 0}^{B}$ vanishes identically, so that Eq. (49) gives

$$
\hat{v}_{r 0}^{A}=\hat{v}_{r 0}^{B}=\hat{v}_{r 0} \equiv 0 .
$$

Therefore, the leading-order solution $\hat{F}_{0}^{\alpha}$ reduces to

$$
\hat{F}_{0}^{\alpha}=\frac{\hat{n}_{0}^{\alpha}}{\left(\pi \hat{T}_{0} / \hat{m}^{\alpha}\right)^{3 / 2}} \exp \left(-\frac{\zeta_{r}^{2}+\left(\zeta_{\theta}-\hat{v}_{\theta 0}\right)^{2}+\zeta_{z}^{2}}{\hat{T}_{0} / \hat{m}^{\alpha}}\right) .
$$

The $m$ th-order equation, Eq. (47), is an inhomogeneous linear integral equation for $\hat{F}_{m}^{\alpha}$. Because of the form of Eq. (52), the left-hand side of Eq. (47) is essentially the linearized collision term for a binary mixture of gases. Therefore, the corresponding homogeneous equation has the independent nontrivial solutions: $\left(a^{\alpha}, \hat{m}^{\alpha} \zeta, \hat{m}^{\alpha} \zeta^{2}\right) \hat{F}_{0}^{\alpha}$, where $a^{\alpha}$ are arbitrary constants, and $\zeta^{2}=\zeta_{r}^{2}+\zeta_{\theta}^{2}+\zeta_{z}^{2}$. In consequence, in order that Eq. (47) has a solution, its inhomogeneous term should satisfy a certain solvability condition, which is reduced to the following form:

$$
\begin{aligned}
& \int \mathcal{D} \hat{F}_{m-1}^{\alpha} \mathrm{d} \zeta=0, \\
& \int \sum_{\alpha=A, B} \hat{m}^{\alpha} \zeta \mathcal{D} \hat{F}_{m-1}^{\alpha} \mathrm{d} \zeta=0, \\
& \int \sum_{\alpha=A, B} \hat{m}^{\alpha} \zeta^{2} \mathcal{D} \hat{F}_{m-1}^{\alpha} \mathrm{d} \zeta=0 .
\end{aligned}
$$

If we insert Eq. (52) in Eqs. (53)-(55) with $m=1$, the $\hat{r}$ component of Eq. (54) gives a constraint shown in Eq. (57) below, but other conditions are satisfied automatically. With this constraint, Eq. (47) with $m=1$ can be solved. The explicit form of the solution $\hat{F}_{1}^{\alpha}$ is given in Appendix B. If we substitute this $\hat{F}_{1}^{\alpha}$ into Eqs. (53)-(55) with $m=2$, we obtain some constraints for the macroscopic variables contained in $\hat{F}_{1}^{\alpha}$. With these constraints, we can proceed to the higher order. In this way, the functional form of $\hat{F}_{n}^{\alpha}$ with respect to $\zeta$ is determined successively $(n=0,1, \ldots)$, and at the same time the constraints on the (unknown) macroscopic variables $h_{n}^{\alpha}$ and $h_{n}$ are obtained in the form of ordinary differential equations. The latter equations are the so-called fluiddynamic equations. The set of equations necessary to determine the leading-order solution $\hat{F}_{0}^{\alpha}$ is essentially obtained from Eq. (53) with $m=2$, the $\hat{r}$ component of Eq. (54) with $m=1$, the $\theta$ component of Eq. (54) with $m=2$, and Eq. (55) with $m=2$, and can be summarized as follows:

$$
\frac{\mathrm{d}}{\mathrm{d} \hat{r}}\left(\hat{r} \hat{\rho}_{0} \hat{v}_{r 1}\right)=0
$$




$$
\begin{aligned}
& \frac{1}{2} \frac{\mathrm{d} \hat{p}_{0}}{\mathrm{~d} \hat{r}}-\frac{\hat{\rho}_{0} \hat{v}_{\theta 0}^{2}}{\hat{r}}=0, \\
& \hat{r} \hat{\rho}_{0} \hat{v}_{r 1} \frac{\mathrm{d}}{\mathrm{d} \hat{r}}\left(\hat{r} \hat{v}_{\theta 0}\right)=\frac{1}{2} \frac{\mathrm{d}}{\mathrm{d} \hat{r}}\left[\hat{r}^{2} \hat{\mu} \hat{T}_{0}^{1 / 2}\left(\frac{\mathrm{d} \hat{v}_{\theta 0}}{\mathrm{~d} \hat{r}}-\frac{\hat{v}_{\theta 0}}{\hat{r}}\right)\right], \\
& \hat{r} \hat{\rho}_{0} \hat{v}_{r 1} \frac{\mathrm{d}}{\mathrm{d} \hat{r}}\left(\hat{v}_{\theta 0}^{2}+\frac{5}{2} \hat{T}_{0}\right)=\frac{\mathrm{d}}{\mathrm{d} \hat{r}}\left[\hat{r} \hat{\mu} \hat{T}_{0}^{1 / 2} \hat{v}_{\theta 0}\left(\frac{\mathrm{d} \hat{v}_{\theta 0}}{\mathrm{~d} \hat{r}}-\frac{\hat{v}_{\theta 0}}{\hat{r}}\right)\right. \\
& \left.+\hat{r} \hat{\lambda} \hat{T}_{0}^{1 / 2} \frac{\mathrm{d} \hat{T}_{0}}{\mathrm{~d} \hat{r}}-k_{T} \frac{\hat{r} \hat{\rho}_{0} \hat{v}_{r 1} \hat{T}_{0}}{\chi_{0}^{A}}\right] \text {, }
\end{aligned}
$$

where

$$
\begin{gathered}
\hat{r} \hat{\rho}_{0} \hat{v}_{r 1}=-\hat{r} \frac{\hat{T}_{0}^{1 / 2}}{\chi_{0}^{B}} \hat{D}_{A B}\left(\frac{\mathrm{d} \chi_{0}^{A}}{\mathrm{~d} \hat{r}}+k_{T} \frac{\mathrm{d} \ln \hat{T}_{0}}{\mathrm{~d} \hat{r}}\right) \\
-2\left(\hat{m}^{B}-\hat{m}^{A}\right) \hat{D}_{A B} \frac{\chi_{0}^{A}}{\hat{T}_{0}^{1 / 2}} \hat{v}_{\theta 0}^{2}, \\
\hat{\rho}_{0}=\hat{m}^{A} \hat{n}_{0}^{A}+\hat{m}^{B} \hat{n}_{0}^{B}, \quad \hat{p}_{0}=\hat{n}_{0} \hat{T}_{0}, \\
\chi_{0}^{\alpha}=\frac{\hat{n}_{0}^{\alpha}}{\hat{n}_{0}}, \quad \hat{n}_{0}=\hat{n}_{0}^{A}+\hat{n}_{0}^{B} .
\end{gathered}
$$

The $\hat{\mu}, \hat{\lambda}, \hat{D}_{A B}$, and $k_{T}\left(=\hat{D}_{T} / \hat{D}_{A B}\right)$ in Eqs. (58)-(60), which are functions of $\hat{T}_{0}$ and $\chi_{0}^{A}$ (or $\chi_{0}^{B}$ ) and depend on the model of molecular interaction, correspond to the viscosity, thermal conductivity, mutual diffusion coefficient, and thermaldiffusion ratio, respectively, where $\hat{D}_{T}$ corresponds to the thermal diffusion coefficient. The definition of these transport coefficients is given in Appendix B. With Eqs. (61a) and (61b), Eqs. (56)-(60) form a closed set of equations for $\hat{n}_{0}^{A}$, $\hat{n}_{0}^{B}, \hat{v}_{r 1}, \hat{v}_{\theta 0}$, and $\hat{T}_{0}$. Equations (56)-(59) are, respectively, the conservation equations for the total mass, the $\hat{r}$ component of the total momentum, its $\theta$ component, and the total energy. Equation (60) expresses the diffusion in the radial direction.

In the derivation of the Hilbert solution, we have used the fact that the radial component of the flow velocity of the noncondensable gas is identically zero, which is a consequence of the boundary condition (30) on the cylinders. Except this, however, the boundary condition has not been taken into account so far. We now consider the boundary condition. Let us assume that the macroscopic variables $\hat{n}_{0}^{\alpha}$, $\hat{v}_{\theta 0}$, and $\hat{T}_{0}$ in $\hat{F}_{0}^{\alpha}$ take the following values on the surfaces of the cylinders; at $\hat{r}=1$,

$$
\hat{n}_{0}^{A}=1, \quad \hat{v}_{\theta 0}=\frac{V_{\mathrm{I}}}{V_{\mathrm{th}}}, \quad \hat{T}_{0}=1,
$$

and at $\hat{r}=L_{\mathrm{II}} / L_{\mathrm{I}}$,

$$
\hat{n}_{0}^{A}=\frac{n_{\mathrm{II}}}{n_{\mathrm{I}}}, \quad \hat{v}_{\theta 0}=\frac{V_{\mathrm{II}}}{V_{\mathrm{th}}}, \quad \hat{T}_{0}=\frac{T_{\mathrm{II}}}{T_{\mathrm{I}}} .
$$

Then, because of relation (33), it is seen that $\hat{F}_{0}^{\alpha}$ satisfies the boundary condition (30) at the leading order (the zeroth or- der of $\epsilon$ ). The restrictions (62) and (63) give the consistent boundary conditions on the cylinders for the fluid-dynamic equations (56)-(61). Note that the boundary conditions (62) and (63) contain only the dimensionless parameters $n_{\mathrm{II}} / n_{\mathrm{I}}$, $T_{\mathrm{II}} / T_{\mathrm{I}}, V_{\mathrm{I}} / V_{\mathrm{th}}$, and $V_{\mathrm{II}} / V_{\mathrm{th}}$, and do not depend on the reference quantity $n_{\mathrm{I}}$ or $T_{\mathrm{I}}$.

The fluid-dynamic system, Eqs. (56)-(63), gives the solution $\hat{n}_{0}^{A}, \hat{n}_{0}^{B}, \hat{v}_{r 1}, \hat{v}_{\theta 0}$, and $\hat{T}_{0}$, with which the leading-order term $\hat{F}_{0}^{\alpha}$ is determined [Eq. (52)]. In this way, we obtain the leading-order solution of the boundary-value problem, Eqs. (17) and (30), by the Hilbert expansion.

The fluid-dynamic type equations for the next order, i.e., for $\hat{n}_{1}^{A}, \hat{n}_{1}^{B}, \hat{v}_{r 2}, \hat{v}_{\theta 1}$, and $\hat{T}_{1}$ (it can be shown that $\hat{T}_{1}^{A}=\hat{T}_{1}^{B}=\hat{T}_{1}$ ), are derived from Eq. (53) with $m=3$, the $\hat{r}$ component of Eq. (54) with $m=2$, the $\theta$ component of Eq. (54) with $m=3$, and Eq. (55) with $m=3$. However, the first-order Hilbert solution (B1) cannot be made to satisfy the boundary condition on the cylinders. Therefore, in order to obtain the first-order solution satisfying the boundary condition, we have to introduce the correction to Eq. (B1) in thin layers with thickness of the order of $\epsilon$ (or of the order of the mean free path $\ell_{c}$ in $r$ ) adjacent to the cylinders (the Knudsen-layer correction). The analysis of the Knudsen-layer correction gives the appropriate boundary conditions for the fluiddynamic type equations for the next order.

\section{B. Continuum limit and the ghost effect}

Here, we give a brief remark on the behavior of the mixture in the continuum limit where Kn or $\epsilon$ vanishes. In this limit, the solution is given by Eq. (52), and the macroscopic variables reduce to their respective leading order terms, i.e., $\left(\hat{n}^{A}, \hat{n}^{B}, \hat{T}, \hat{v}_{\theta}, \hat{v}_{r}\right) \rightarrow\left(\hat{n}_{0}^{A}, \hat{n}_{0}^{B}, \hat{T}_{0}, \hat{v}_{\theta 0}, 0\right) \quad$ [cf. Eq. (51)]. That is, in this limit, evaporation and condensation of the vapor stop, and the radial component of the flow velocity $\hat{v}_{r}$ vanishes. Therefore, we might expect that the flow field in this limit is given by the flow field in the same limit of the ordinary cylindrical Couette flow of a binary mixture of noncondensable gases (or between impermeable cylinders). However, this is not correct, and the situation is more involved. The leading-order terms $\hat{n}_{0}^{A}, \hat{n}_{0}^{B}, \hat{T}_{0}$, and $\hat{v}_{\theta 0}$ are determined together with the first-order term $\hat{v}_{r 1}$ of the radial component of the flow velocity. This means that, in spite of the fact that there is no evaporation or condensation, the flow field in the continuum limit is affected by the infinitesimal radial flow (or infinitesimal evaporation and condensation) [note that $\hat{v}_{r}=\hat{v}_{r 1} \epsilon+\cdots \rightarrow 0$, but $\hat{v}_{r 1}$ is of $\left.O(1)\right]$. This is an example of the ghost effect (see the last paragraph). In particular, the situation in the present problem is essentially the same as that of the plane Couette flow of the mixture of a vapor and a noncondensable gas. ${ }^{26}$ Some numerical examples of the continuum limit obtained from the fluiddynamic system, Eqs. (56)-(63), will be given in Sec. IV.

In the case of the ordinary cylindrical Couette flow of a binary mixture of noncondensable gases, i.e., in the case where the $A$ component is also a noncondensable gas, the fluid-dynamic system can be derived readily by a slight modification of the analysis in the present section. The re- 

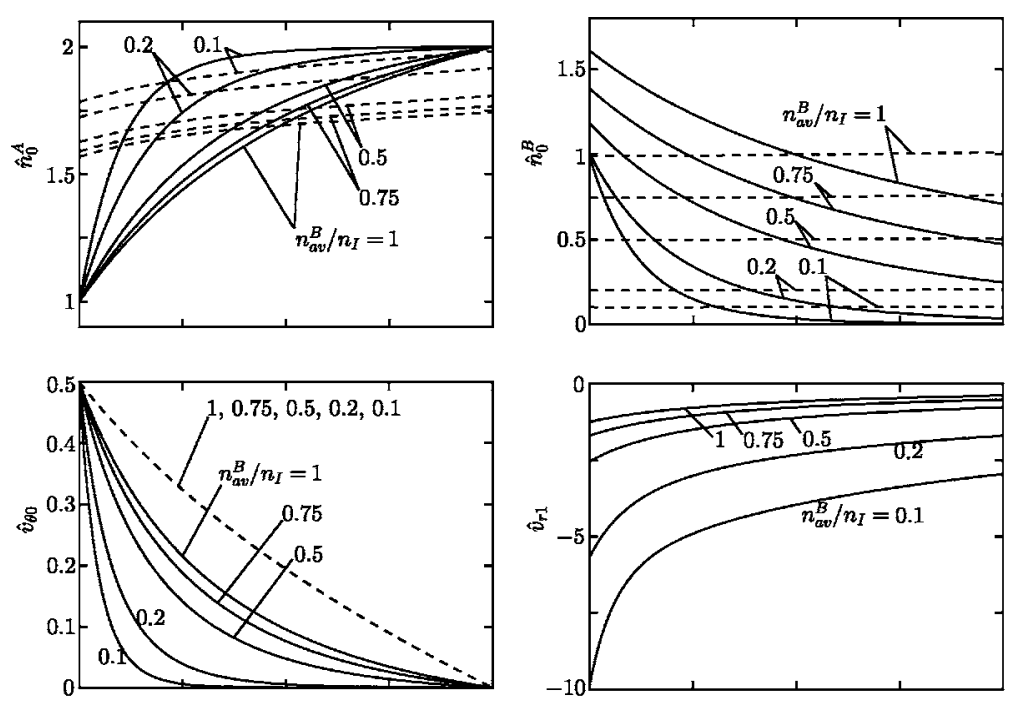

Phys. Fluids 18, 087103 (2006)

FIG. 1. Profiles of the macroscopic quantities in the continuum limit for various $n_{\mathrm{av}}^{B} / n_{\mathrm{I}}$ (hard-sphere molecules) in the case of $n_{\mathrm{II}} / n_{\mathrm{I}}=2, V_{\mathrm{I}} / V_{\mathrm{th}}=0.5, \mathrm{~m}^{B} / \mathrm{m}^{A}$ $=0.2$, and $d^{B} / d^{A}=1 \quad\left(L_{\mathrm{II}} / L_{\mathrm{I}}=2, T_{\mathrm{II}} / T_{\mathrm{I}}=1\right.$, and $\left.V_{\mathrm{II}}=0\right)$. The dashed line indicates the corresponding results for ordinary cylindrical Couette flow (see the main text for the meaning of $n_{\mathrm{I}}$ in this case).
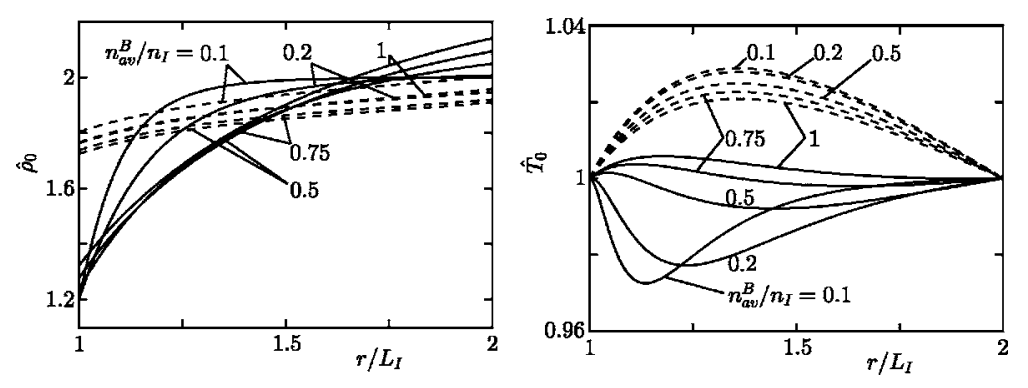

sulting fluid-dynamic equations are Eqs. (56)-(61) with $\hat{v}_{r 1}=0$, and the boundary conditions are Eqs. (62) and (63) with the conditions for $\hat{n}_{0}^{A}$ discarded. In this system, $n_{\mathrm{I}}$, which appears in the nondimensionalization (16), should be regarded as an appropriate reference molecular number density, and $n_{\mathrm{II}}$ disappears. In addition, we have to specify a parameter associated with the amount of the $A$ component, say $n_{\mathrm{av}}^{A} / n_{\mathrm{I}}$ with $n_{\mathrm{av}}^{A}$ being the average molecular number density of the $A$ component. Some numerical solution of this system (ordinary cylindrical Couette flow, for short) will also be shown in the next section.

Finally we comment on the ghost effect mentioned above. The problems of rarefied gas dynamics generally contain various parameters, such as the Mach number, Froude number, and accommodation coefficients, in addition to the Knudsen number. If we consider the continuum limit in the situation where one of the other parameters vanishes together with the Knudsen number, then the effect of the parameter may remain finite in the continuum limit though the parameter itself vanishes. Such an effect, caused by an infinitesimal parameter, in the continuum limit is called the ghost effect. The vanishing parameter can be chosen either artificially by ourselves or naturally by the problem. For example, if we consider a stationary gas in a closed container at rest with an arbitrary temperature distribution (we neglect the gravity), a flow with Mach number of the order of the Knudsen number occurs in general when the Knudsen number is small. This flow, which vanishes in the continuum limit, has a finite effect on the temperature distribution in the gas. Therefore, contrary to common belief, the steady heat-conduction equation (or more generally, the Navier-Stokes system) fails to describe the temperature distribution even in the continuum limit. This fact was pointed out and clarified in Ref. 19, where the correct fluid-dynamic equations and their boundary conditions for the temperature field were derived systematically from the Boltzmann equation and its kinetic boundary condition, and some concrete examples demonstrating the effect were presented (the possibility of invalidity of the heat-conduction equation was also noted in Ref. 43). In this example, the other vanishing parameter, which is naturally chosen by the problem, is the Mach number. In the problem studied in the present paper, the infinitesimal parameter is the Mach number based on the radial component of the flow velocity. The ghost effect has been investigated extensively in the papers following Ref. 19, and several interesting and nonintuitive phenomena have been clarified (see Refs. 18 and 20-25 and the references in Refs. 22 and 25; for the case of a gas mixture, we refer to Refs. 26 and 44-46). The most recent discovery is a surprising effect caused by an infinitesimal curvature of the boundary in plane Couette flow. ${ }^{24,25}$

\section{RESULTS AND DISCUSSIONS}

\section{A. Numerical analysis}

In Secs. IV B and IV C, we show some numerical results and discuss the behavior of the vapor and noncondensable gas in the continuum limit. The main result that will be presented here is the result of numerical analysis based on the fluid-dynamic system derived in Sec. III. We assume that both components consist of hard-sphere molecules. Then, the database for the transport coefficients $\hat{\mu}, \hat{\lambda}, \hat{D}_{A B}$, and $k_{T}$ oc- 

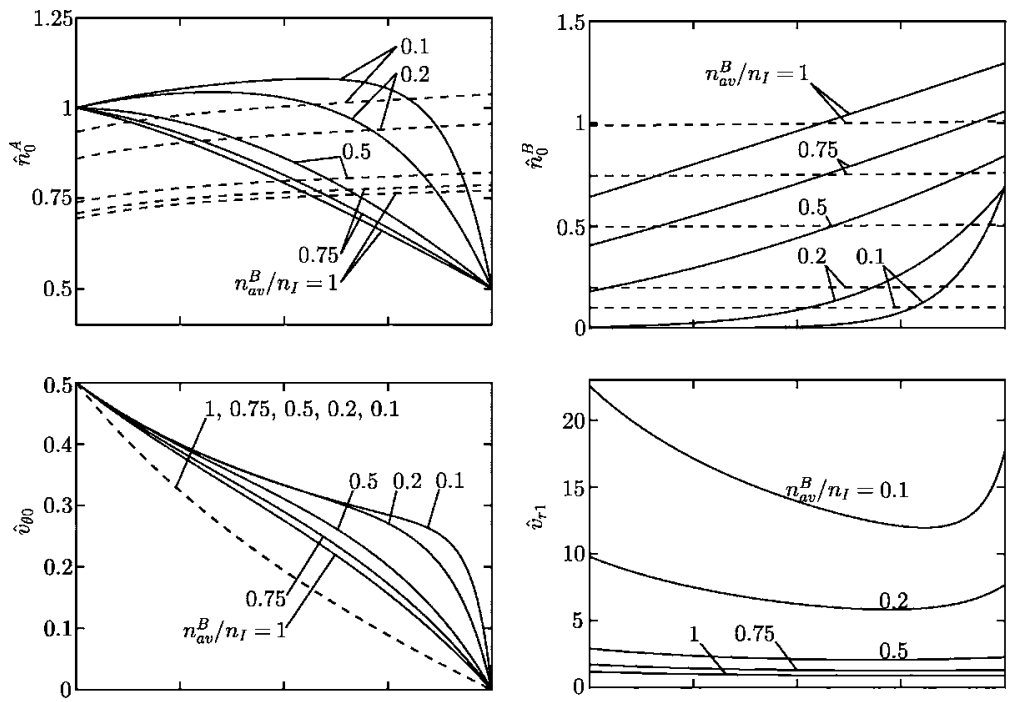

FIG. 2. Profiles of the macroscopic quantities in the continuum limit for various $n_{\mathrm{av}}^{B} / n_{\mathrm{I}}$ (hard-sphere molecules) in the case of $n_{\mathrm{II}} / n_{\mathrm{I}}=0.5, V_{\mathrm{I}} / V_{\mathrm{th}}=0.5, \mathrm{~m}^{B} / \mathrm{m}^{A}$ $=0.2$, and $d^{B} / d^{A}=1\left(L_{\mathrm{II}} / L_{\mathrm{I}}=2, T_{\mathrm{II}} / T_{\mathrm{I}}=1\right.$, and $\left.V_{\mathrm{II}}=0\right)$. See the caption of Fig. 1.
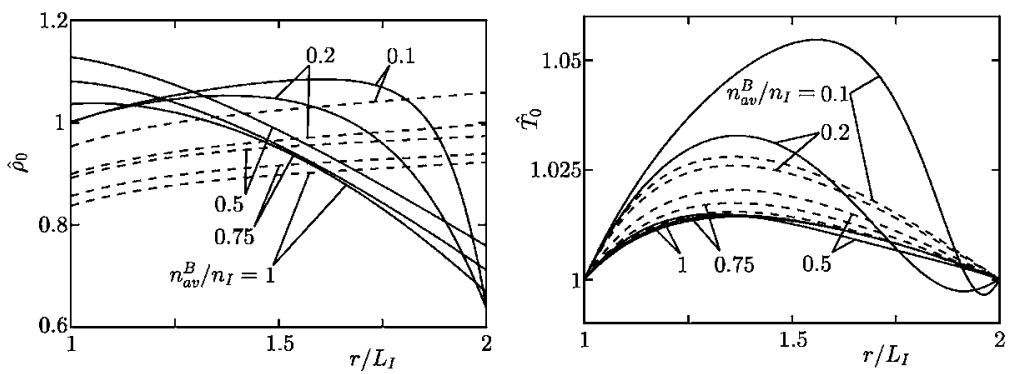

curring in Eqs. (58)-(60), which gives their values for arbitrary values of the concentration $\chi_{0}^{A}$, is available ${ }^{47}$ (note that they are independent of $\hat{T}_{0}$ for hard-sphere molecules). The ordinary differential equations (56)-(60) with the boundary conditions (62) and (63) have been solved numerically by an iterative finite-difference method. Since Eqs. (62) and (63) do not depend on $n_{\mathrm{I}}$ or $T_{\mathrm{I}}$ itself, the problem is characterized by the dimensionless parameters $n_{\mathrm{II}} / n_{\mathrm{I}}, T_{\mathrm{II}} / T_{\mathrm{I}}, V_{\mathrm{I}} / V_{\mathrm{th}}$, $V_{\mathrm{II}} / V_{\mathrm{th}}, n_{\mathrm{av}}^{B} / n_{\mathrm{I}}, L_{\mathrm{II}} / L_{\mathrm{I}}, m^{B} / m^{A}$, and $d^{B} / d^{A}$ for hard-sphere molecules. We let

$$
\frac{T_{\mathrm{II}}}{T_{\mathrm{I}}}=1, \quad \frac{V_{\mathrm{II}}}{V_{\mathrm{th}}}=0, \quad \frac{L_{\mathrm{II}}}{L_{\mathrm{I}}}=2,
$$

throughout Secs. IV B and IV C. The relations between $\hat{n}_{0}^{\alpha}$, $\hat{v}_{\theta 0}, \hat{T}_{0}$, etc., occurring in the fluid-dynamic system and the physical quantities in the continuum limit are as follows:

$$
\begin{aligned}
& \hat{n}_{0}^{\alpha}=\frac{n^{\alpha}}{n_{\mathrm{I}}}, \quad \hat{\rho}_{0}=\frac{\rho}{m^{A} n_{\mathrm{I}}}, \\
& \hat{T}_{0}=\frac{T}{T_{\mathrm{I}}}, \quad \hat{v}_{\theta 0}=\frac{v_{\theta}}{V_{\mathrm{th}}}, \\
& \hat{v}_{r 1}=\lim _{\epsilon \rightarrow 0} \frac{v_{r}}{V_{\mathrm{th}}} \frac{1}{\epsilon}=\lim _{\mathrm{Kn} \rightarrow 0} \frac{v_{r}}{V_{\mathrm{th}}} \frac{2}{\sqrt{\pi} \mathrm{Kn}},
\end{aligned}
$$

$$
\frac{2}{\left(L_{\mathrm{II}} / L_{\mathrm{I}}\right)^{2}-1} \int_{1}^{L_{\mathrm{II}} / L_{\mathrm{I}}} \hat{r} \hat{n}_{0}^{B} \mathrm{~d} \hat{r}=\frac{n_{\mathrm{av}}^{B}}{n_{\mathrm{I}}} .
$$

In addition to the numerical analysis for the fluiddynamic system, we have also performed a Monte Carlo simulation for the original Boltzmann system, Eqs. (17) and (30), for small Knudsen numbers, using the direct simulation Monte Carlo (DSMC) method. ${ }^{48,49}$ We have assumed hardsphere molecules for both components. We have also assumed the complete condensation condition for the vapor and the diffuse reflection condition for the noncondensable gas, i.e., Eq. (30) with Eqs. (35)-(38).

\section{B. Ghost effect}

In Figs. 1 and 2, we show, by solid lines, the profiles of the macroscopic quantities $\hat{n}_{0}^{A}, \hat{n}_{0}^{B}, \hat{v}_{\theta 0}, \hat{v}_{r 1}, \hat{\rho}_{0}$, and $\hat{T}_{0}$, obtained from Eqs. (56)-(63), for various values of $n_{\mathrm{av}}^{B} / n_{\mathrm{I}}$ in the case of $V_{\mathrm{I}} / V_{\text {th }}=0.5, m^{B} / m^{A}=0.2$, and $d^{B} / d^{A}=1$ : Fig. 1 is for $n_{\mathrm{II}} / n_{\mathrm{I}}=2$, and Fig. 2 for $n_{\mathrm{II}} / n_{\mathrm{I}}=0.5$. In Fig. 1, infinitesimal evaporation takes place on the outer cylinder and infinitesimal condensation on the inner, so that there is an infinitesimal inward flow $\left(\hat{v}_{r 1}<0\right)$. In Fig. 2, the situation is opposite $\left(\hat{v}_{r 1}>0\right)$. The magnitude of the infinitesimal radial flow $\left(\left|\hat{v}_{r 1} \epsilon\right|\right)$ increases as the amount of the noncondensable gas decreases, i.e., as $n_{\text {av }}^{B} / n_{\mathrm{I}}$ decreases. Correspondingly, the profile of the tangential velocity component $\left(\hat{v}_{\theta 0}\right)$ is pushed inward (Fig. 1) or outward (Fig. 2) with the decrease of $n_{\mathrm{av}}^{B} / n_{\mathrm{I}}$. In Figs. 1 and 2, the results for the ordinary cylin- 

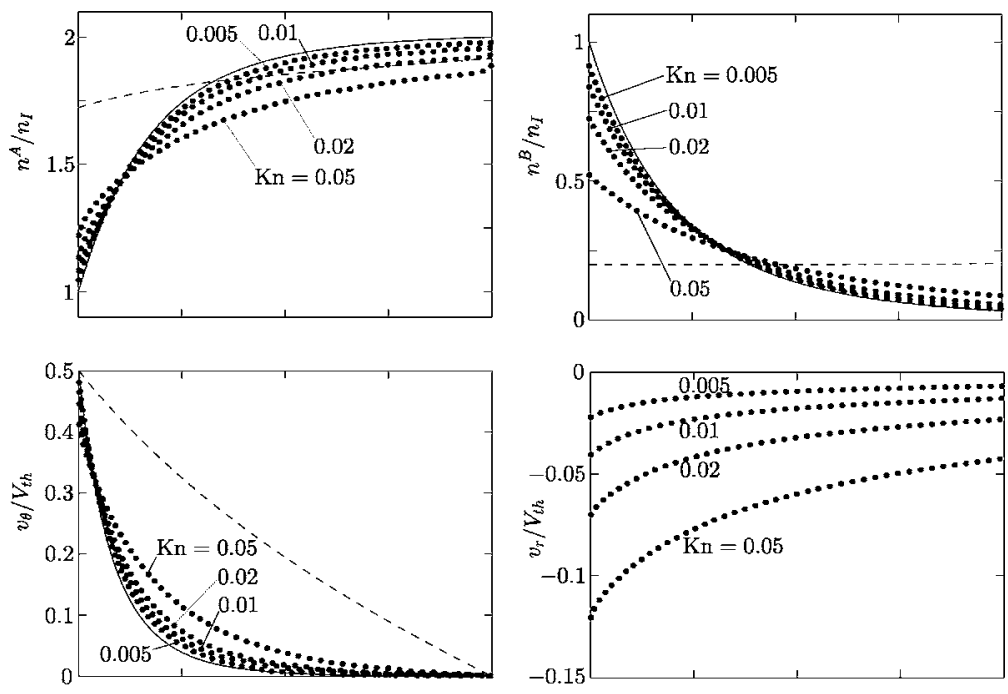

FIG. 3. Profiles of the macroscopic quantities for small $\mathrm{Kn}$ (DSMC result for hard-sphere molecules) in the case of $n_{\mathrm{av}}^{B} / n_{\mathrm{I}}=0.2, n_{\mathrm{II}} / n_{\mathrm{I}}=2, V_{\mathrm{I}} / V_{\mathrm{th}}=0.5, \mathrm{~m}^{B} / \mathrm{m}^{A}=0.2$, and $d^{B} / d^{A}=1 \quad\left(L_{\mathrm{II}} / L_{\mathrm{I}}=2, T_{\mathrm{II}} / T_{\mathrm{I}}=1\right.$, and $\left.V_{\mathrm{II}}=0\right)$. The solid line indicates the profile of the continuum limit, and the dashed line that of the same limit for the ordinary cylindrical Couette flow.
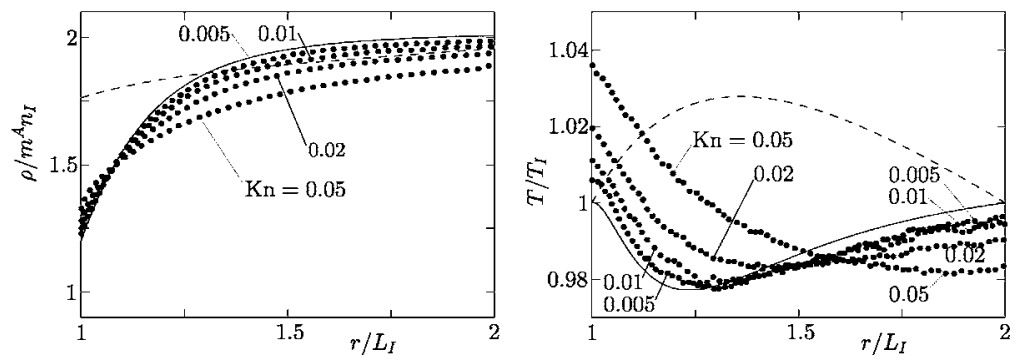

drical Couette flow (i.e., the case where the $A$ component is also noncondensable) are also shown by dashed lines for the corresponding parameters, $V_{\mathrm{I}} / V_{\mathrm{th}}=0.5, \mathrm{~m}^{B} / \mathrm{m}^{A}=0.2$, and $d^{B} / d^{A}=1$. Here, the reference molecular number density $n_{\mathrm{I}}$ has been determined in such a way that the total number of the $A$ component, $n_{\mathrm{I}} \int_{1}^{2} \hat{r} \hat{n}_{0}^{A} \mathrm{~d} \hat{r}$, takes the same value as the corresponding case where the $A$ component is the vapor (see the second paragraph of Sec. III B). The $\hat{v}_{\theta 0}$ for the ordinary cylindrical Couette flow is practically independent of $n_{\text {av }}^{B} / n_{\mathrm{I}}$, i.e., the concentration of the components.

We should now recall that there is no evaporation or condensation (i.e., the radial flow is infinitesimal) in the case of the mixture of a vapor and a noncondensable gas. However, the behavior is quite different from that of the ordinary cylindrical Couette flow. For example, the tangential velocity profile deviates significantly, and the deviation increases as $n_{\mathrm{av}}^{B} / n_{\mathrm{I}}$ decreases. This deviation is due to the ghost effect caused by the infinitesimal evaporation and condensation.

Figures 3 and 4 show the result of the Monte Carlo simulation by the DSMC method: The profiles of $n^{A}, n^{B}, v_{\theta}$, $v_{r}, \rho$, and $T$ are shown for small Knudsen numbers, $\mathrm{Kn}=0.05,0.02,0.01$, and 0.005 . Figure 3 corresponds to the case of Fig. 1 with $n_{\text {av }}^{B} / n_{\mathrm{I}}=0.2$, and Fig. 4 corresponds to the case of Fig. 2 with $n_{\text {av }}^{B} / n_{\mathrm{I}}=0.2$. As $\mathrm{Kn}$ is reduced, the magnitude of the radial component $v_{r}$ decreases, showing the tendency that it vanishes in the limit $\mathrm{Kn} \rightarrow 0$. This is consistent with the fact that $\hat{v}_{r 0}=0$ in the asymptotic analysis. The profiles approach the solid curves that indicate the solution of the fluid-dynamic system. By the way, the dashed lines indicate the solution of the fluid-dynamic system for the ordinary cylindrical Couette flow.
The DSMC computation is seemingly easy in the present spatially one-dimensional problem, but in reality it is not an easy task because of the structure of the flow. When the Knudsen number is small, the $\mathrm{Kn}^{0}$-order quantities such as $\hat{v}_{\theta 0}$ are determined together with the Kn-order radial velocity $\hat{v}_{r 1} \epsilon$. Therefore, in the DSMC computation, we need to obtain the small radial velocity precisely in order to describe the other macroscopic quantities correctly. As is well known, however, one of the drawbacks of the DSMC method is the difficulty in obtaining small quantities because they are buried in the statistical fluctuations inherent to the method. Since the radial velocity in the present problem decreases in proportion to the Knudsen number, the computation becomes increasingly difficult with the decrease of the Knudsen number. This situation has been pointed out in the study of the two-surface problems of a vapor-gas mixture. ${ }^{26}$ To overcome this difficulty, a rather large computational system and a long-time run are inevitable. In the present computation, the interval $1 \leqslant r / L_{\mathrm{I}} \leqslant 2$ is divided into 400 uniform cells $(\mathrm{Kn}=0.05, \quad 0.02$, and 0.01$)$ or 800 uniform cells $(\mathrm{Kn}=0.005) ; 100$ simulation particles per cell are used for the number density $n^{A}=n_{\mathrm{I}}$ in Fig. 3, and 200 particles per cell for $n^{A}=n_{\mathrm{I}}$ in Fig. 4. The time step $\Delta t$ is $4.51 \times 10^{-3} t_{c}$ $(\mathrm{Kn}=0.05), 5.64 \times 10^{-3} t_{c} \quad(\mathrm{Kn}=0.02$ and 0.01$)$, or 1.13 $\times 10^{-2} t_{c}(\mathrm{Kn}=0.005)$, where $t_{c}$ is the mean free time corresponding to $T_{\mathrm{I}}$ and $\ell_{c}$. The computation was performed over $10^{6} \Delta t \quad(\mathrm{Kn}=0.05), \quad 2 \times 10^{6} \Delta t \quad(\mathrm{Kn}=0.02), \quad$ or $4 \times 10^{6} \Delta t$ $(\mathrm{Kn}=0.01$ and 0.005$)$ after the steady state has been established, and the averages over these time intervals are shown in the figures. 

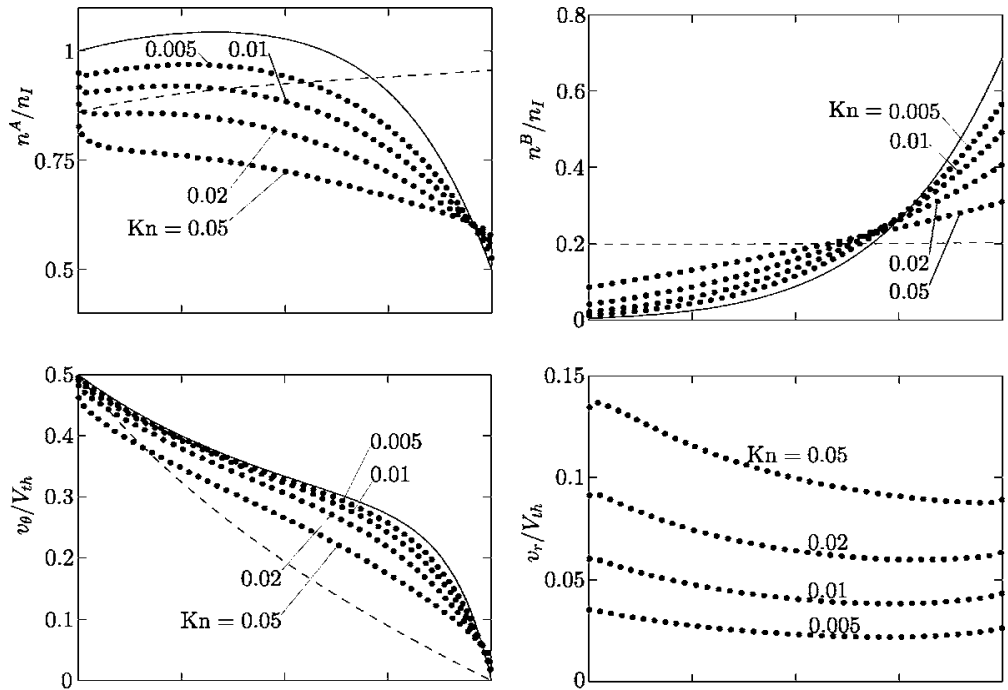

FIG. 4. Profiles of the macroscopic quantities for small $\mathrm{Kn}$ (DSMC result for hard-sphere molecules) in the case of $n_{\mathrm{av}}^{B} / n_{\mathrm{I}}=0.2, n_{\mathrm{II}} / n_{\mathrm{I}}=0.5, V_{\mathrm{I}} / V_{\mathrm{th}}=0.5, \mathrm{~m}^{B} / \mathrm{m}^{A}$ $=0.2$, and $d^{B} / d^{A}=1\left(L_{\mathrm{II}} / L_{\mathrm{I}}=2, T_{\mathrm{II}} / T_{\mathrm{I}}=1\right.$, and $\left.V_{\mathrm{II}}=0\right)$. See the caption of Fig. 3 .
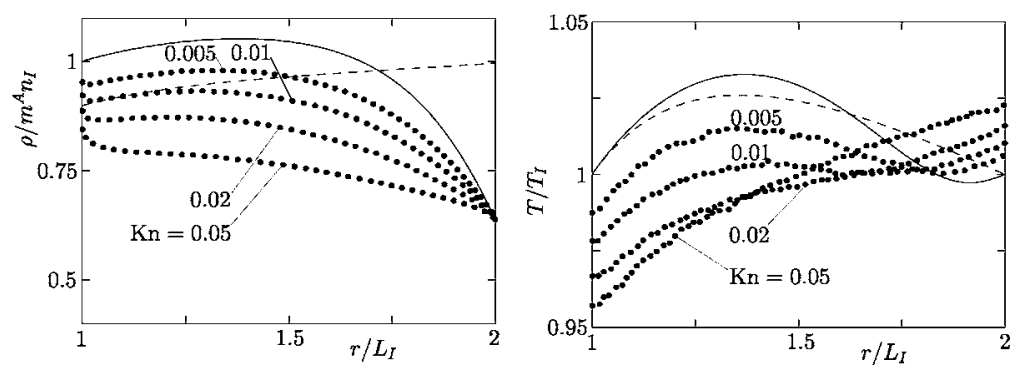

\section{Bifurcation}

Let us denote by $M_{r}$ the mass flow of the vapor in the radial direction per unit time and per unit length of the axial coordinate $z$, which is infinitesimal in the continuum limit. Then, it is related to $\hat{\rho}_{0} \hat{v}_{r 1}$ and $\hat{m}^{A} \hat{n}_{0}^{A} \hat{v}_{r 1}^{A}$ as

$$
\begin{aligned}
& \hat{r} \hat{\rho}_{0} \hat{v}_{r 1}=\hat{r} \hat{m}^{A} \hat{n}_{0}^{A} \hat{v}_{r 1}^{A}=\lim _{\mathrm{Kn} \rightarrow 0} \tilde{M}, \\
& \tilde{M}=\frac{M_{r}}{2 \pi L_{\mathrm{I}} m^{A} n_{\mathrm{I}} V_{\mathrm{th}}} \frac{2}{\sqrt{\pi} \mathrm{Kn}},
\end{aligned}
$$

where $\tilde{M}$ is introduced for the later convenience.

Figure 5 shows $\hat{r} \hat{\rho}_{0} \hat{v}_{r 1}$ vs $V_{\mathrm{I}} / V_{\mathrm{th}}$, obtained from Eqs. (56)-(63), for various values of $n_{\mathrm{av}}^{B} / n_{\mathrm{I}}$ in the case of $n_{\mathrm{II}} / n_{\mathrm{I}}$ $=1.5, m^{B} / m^{A}=1$, and $d^{B} / d^{A}=1 \quad[(\mathrm{a})]$ and $n_{\mathrm{II}} / n_{\mathrm{I}}=1.2$, $\mathrm{m}^{B} / \mathrm{m}^{A}=0.2$, and $d^{B} / d^{A}=1[(\mathrm{~b})]$. In Fig. 5(a), as $n_{\mathrm{av}}^{B} / n_{\mathrm{I}}$ de- creases from 1 to 0.1 , the curve becomes steep near $V_{\mathrm{I}} / V_{\text {th }}$ $=1$, and the gradient of the curve there becomes negative with the further decrease of $n_{\mathrm{av}}^{B} / n_{\mathrm{I}}$. For example, for $n_{\mathrm{av}}^{B} / n_{\mathrm{I}}$ $=0.05$, there are three values of $\hat{r} \hat{\rho}_{0} \hat{v}_{r 1}$ at the same $V_{\mathrm{I}} / V_{\text {th }}$ in a certain interval. In other words, the solution is not unique, and the bifurcation of the solution takes place. As $n_{\mathrm{av}}^{B} / n_{\mathrm{I}}$ is decreased further, the curve tends to approach the dot-dashed curve that indicates the pure-vapor case. ${ }^{18} \mathrm{~A}$ basically similar tendency, with bifurcation at smaller $V_{\mathrm{I}} / V_{\text {th }}$, is observed in Fig. 5(b). It can be shown from Eqs. (56)-(60) that in Fig. 5 (a) all the curves pass a single point, $\left(V_{\mathrm{I}} / V_{\mathrm{th}}, \hat{r} \hat{\rho}_{0} \hat{v}_{r 1}\right)$ $=(1.0053,0)$, because of the special choice $\mathrm{m}^{B} / \mathrm{m}^{A}=d^{B} / d^{A}$ $=1$. The same is seemingly true in Fig. 5(b), but a close examination of the numerical result shows that the curves do not pass a single point.

Figure 6(a) shows a different aspect of Fig. 5(a), that is,

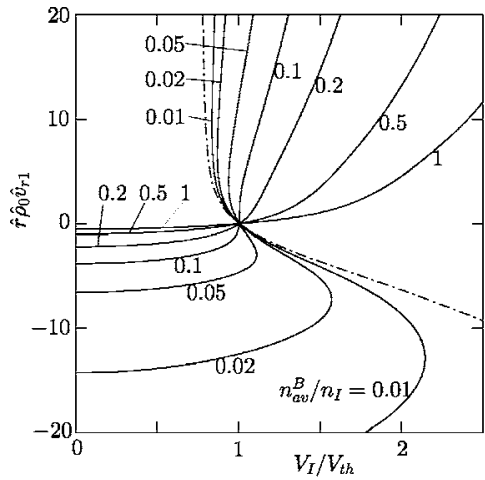

(a)

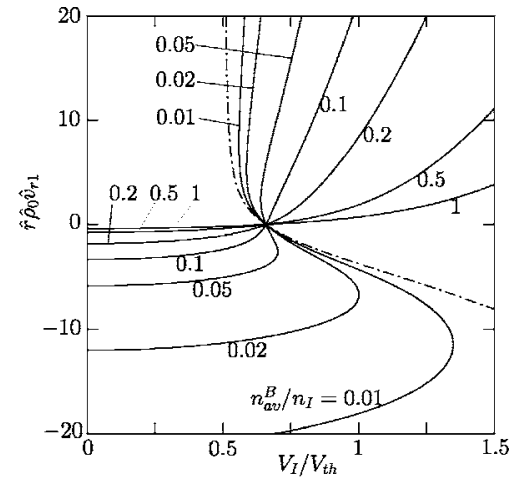

(b)
FIG. 5. The $\hat{r} \hat{\rho}_{0} \hat{v}_{r 1}$ vs $V_{\mathrm{I}} / V_{\text {th }}$ in the continuum limit for various $n_{\mathrm{av}}^{B} / n_{\mathrm{I}}$ (hard-sphere molecules) $\left(L_{\mathrm{II}} / L_{\mathrm{I}}=2\right.$, $T_{\mathrm{II}} / T_{\mathrm{I}}=1$, and $V_{\mathrm{II}}=0$ ). (a) $n_{\mathrm{II}} / n_{\mathrm{I}}=1.5, \mathrm{~m}^{B} / \mathrm{m}^{A}=1$, and $d^{B} / d^{A}=1$, (b) $n_{\mathrm{II}} / n_{\mathrm{I}}=1.2, \mathrm{~m}^{B} / \mathrm{m}^{A}=0.2$, and $d^{B} / d^{A}=1$. The dot-dashed line indicates the pure vapor case (Ref. 18) 


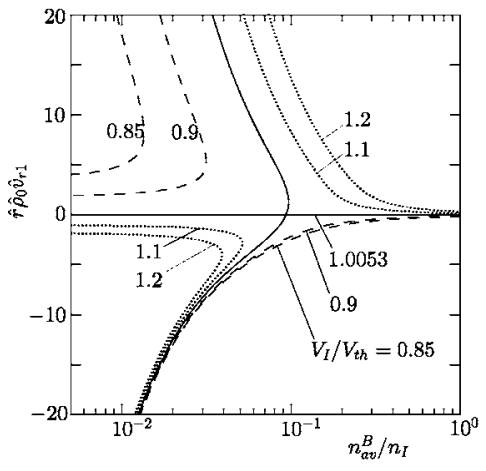

(a)
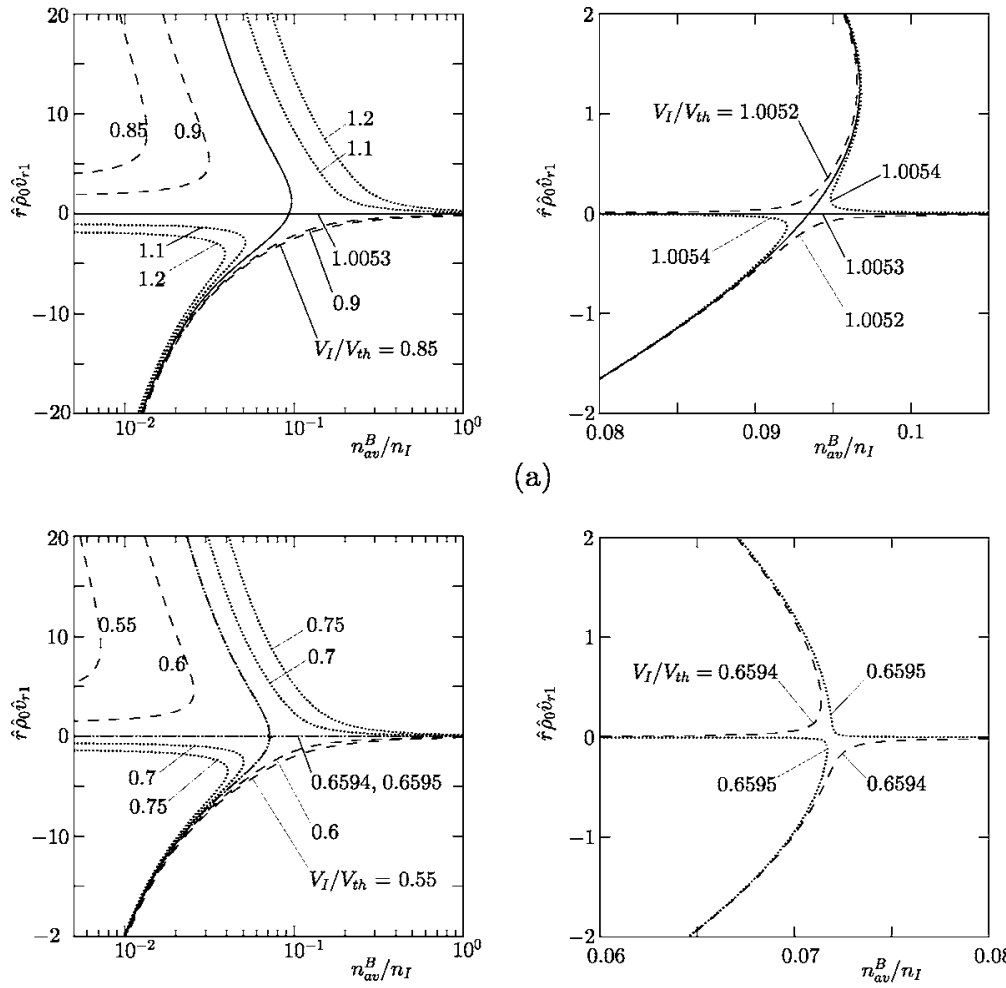

(b)

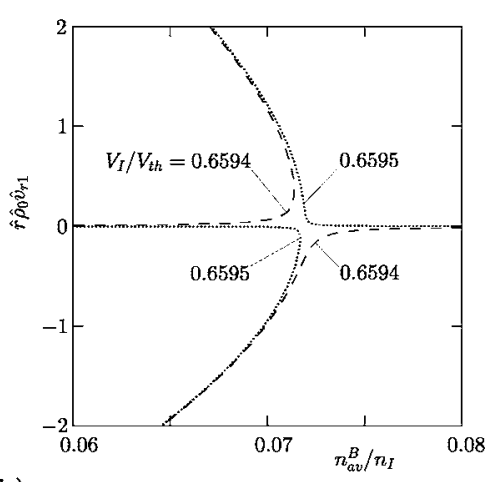

FIG. 6. The $\hat{r} \hat{\rho}_{0} \hat{v}_{r 1}$ vs $n_{\mathrm{av}}^{B} / n_{\mathrm{I}}$ in the continuum limit for various $V_{\mathrm{I}} / V_{\text {th }}$ (hard-sphere molecules) $\left(L_{\mathrm{II}} / L_{\mathrm{I}}=2\right.$, $T_{\mathrm{II}} / T_{\mathrm{I}}=1$, and $V_{\mathrm{II}}=0$ ). (a) $n_{\mathrm{II}} / n_{\mathrm{I}}=1.5, \mathrm{~m}^{B} / \mathrm{m}^{A}=1$, and $d^{B} / d^{A}=1$, (b) $n_{\mathrm{II}} / n_{\mathrm{I}}=1.2, m^{B} / m^{A}=0.2$, and $d^{B} / d^{A}=1$. $\hat{r} \hat{\rho}_{0} \hat{v}_{r 1}$ vs $n_{\text {av }}^{B} / n_{\mathrm{I}}$ is plotted for different values of $V_{\mathrm{I}} / V_{\text {th }}$ in the case of $n_{\mathrm{II}} / n_{\mathrm{I}}=1.5, \mathrm{~m}^{B} / \mathrm{m}^{A}=1$, and $d^{B} / d^{A}=1$. The right-hand figure is a magnified one. As $V_{\mathrm{I}} / V_{\text {th }}$ increases from 0.85 , two branches indicated by the dashed line approach, and, as shown by the solid line, the recombination of the two branches takes place at $V_{\mathrm{I}} / V_{\mathrm{th}}=1.0053$, namely, at the fixed point in Fig. 5(a). Then, the new branches, indicated by the dotted line, separate with the further increase of $V_{\mathrm{I}} / V_{\mathrm{th}}$. Figure 6(b) is the corresponding figure for Fig. 5(b). In this case, the recombination takes place at a value of $V_{\mathrm{I}} / V_{\text {th }}$ between
0.6594 and 0.6595 (the curves at these two values are shown by the dashed and dotted lines, respectively, but they almost coincide in the left-hand figure).

In Fig. 7(a), the profiles of $\hat{v}_{\theta 0}$ and $\hat{v}_{r 1}$ of the three bifurcated solutions at $V_{\mathrm{I}} / V_{\mathrm{th}}=1.0053$ are shown for three different values of $n_{\mathrm{av}}^{B} / n_{\mathrm{I}}$, i.e., 0.08, 0.05, and 0.02 [see Figs. 5(a) and 6(a)]. The solid line indicates the solution with negative $\hat{v}_{r 1}$ (in the sense that $\hat{v}_{r 1}<c_{1}<0$ with a finite $c_{1}$ ), the dot-dashed line that with positive $\hat{v}_{r 1}$ (in the sense that $0<c_{2}<\hat{v}_{r 1}$ with a finite $c_{2}$ ), and the dashed line that with

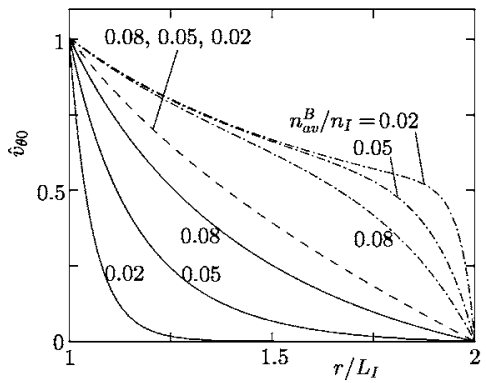

(a)
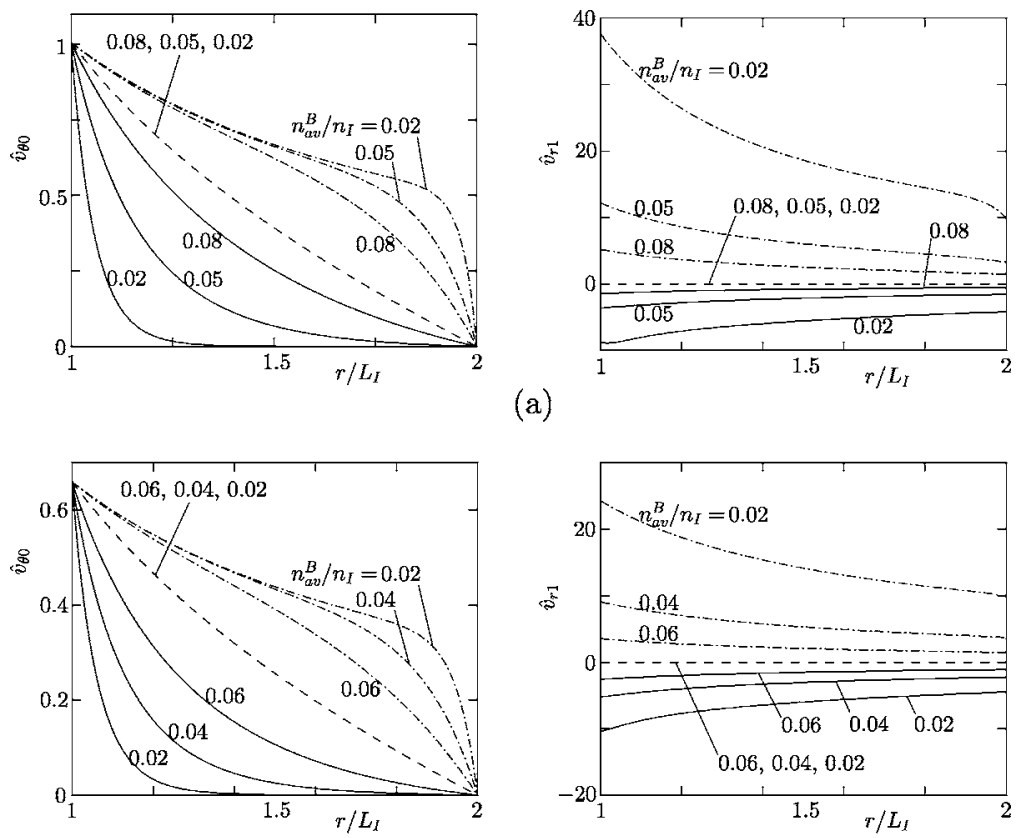

FIG. 7. Profiles of $\hat{v}_{\theta 0}$ and $\hat{v}_{r 1}$ of the three bifurcated solutions in the continuum limit (hard-sphere molecules) $\left(L_{\mathrm{II}} / L_{\mathrm{I}}=2, T_{\mathrm{II}} / T_{\mathrm{I}}=1\right.$, and $\left.V_{\mathrm{II}}=0\right)$. (a) At $V_{\mathrm{I}} / V_{\text {th }}$ $=1.0053$ for $n_{\mathrm{aV}}^{B} / n_{\mathrm{I}}=0.08,0.05$, and 0.02 in the case of $n_{\mathrm{II}} / n_{\mathrm{I}}=1.5, m^{B} / m^{A}=1$, and $d^{B} / d^{A}=1$ [cf. Figs. 5(a) and 6(a)], (b) at $V_{\mathrm{I}} / V_{\text {th }}=0.6595$ for $n_{\mathrm{av}}^{B} / n_{\mathrm{I}}=0.06,0.04$, and 0.02 in the case of $n_{\mathrm{II}} / n_{\mathrm{I}}=1.2, \mathrm{~m}^{B} / \mathrm{m}^{A}=0.2$, and $d^{B} / d^{A}$ $=1$ [cf. Figs. 5(b) and 6(b)]. The solid line indicates the solution with negative and finite $\hat{v}_{r 1}$, the dot-dashed line that with positive and finite $\hat{v}_{r 1}$, and the dashed line that with $\hat{v}_{r 1}=0$ in (a) and $\hat{v}_{r 1} \simeq 0$ in (b). 


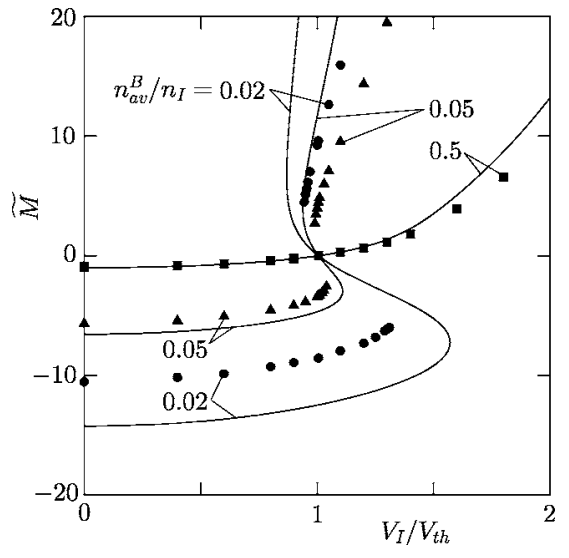

FIG. 8. Magnified mass flow rate $\tilde{M}$ vs $V_{\mathrm{I}} / V_{\text {th }}$ at $\mathrm{Kn}=0.005$ (DSMC result for hard-sphere molecules) for $n_{\mathrm{av}}^{B} / n_{\mathrm{I}}=0.5(\mathbf{\square}), 0.05(\mathbf{\Delta})$, and $0.02(\mathbf{\bullet})$ in the case of $n_{\mathrm{II}} / n_{\mathrm{I}}=1.5, \mathrm{~m}^{B} / \mathrm{m}^{A}=1$, and $d^{B} / d^{A}=1\left(L_{\mathrm{II}} / L_{\mathrm{I}}=2, T_{\mathrm{II}} / T_{\mathrm{I}}=1\right.$, and $\left.V_{\text {II }}=0\right)$. The solid line indicates the results in the continuum limit.

$\hat{v}_{r 1}=0$, which is common to all $n_{\mathrm{av}}^{B} / n_{\mathrm{I}}$ and corresponds to the fixed point in Fig. 5(a) and to the ordinary cylindrical Couette flow. Figure 7(b) is the corresponding figure for the three bifurcated solutions at $V_{\mathrm{I}} / V_{\text {th }}=0.6595$ for three different values of $n_{\text {av }}^{B} / n_{\mathrm{I}}$, i.e., 0.06, 0.04, and 0.02 [see Figs. 5(b) and 6(b)]. The three different types of lines have the same meaning as in Fig. 7(a), except that the dashed line indicates the solution with very small $\left|\hat{v}_{r 1}\right|\left(\simeq 10^{-3}\right)$. This solution changes very slightly with $n_{\text {av }}^{B} / n_{\mathrm{I}}$ though invisible from the figure.

In Figs. 8 and 9, we show some results obtained by the DSMC method for the original Boltzmann system. Figure 8 shows the results for the case corresponding to Fig. 5(a). More specifically, the symbols $\boldsymbol{\square}, \boldsymbol{\Delta}$, and $\boldsymbol{\bullet}$ indicate $\tilde{M}$ in Eq. (67) vs $V_{\mathrm{I}} / V_{\text {th }}$ for $n_{\text {av }}^{B} / n_{\mathrm{I}}=0.5,0.05$, and 0.02 at $\mathrm{Kn}$ $=0.005$ in the case of $n_{\mathrm{II}} / n_{\mathrm{I}}=1.5, \mathrm{~m}^{B} / \mathrm{m}^{A}=1$, and $d^{B} / d^{A}=1$. The corresponding curve in the continuum limit based on the fluid-dynamic system is also shown for comparison. The DSMC result demonstrates the nonuniqueness of the solution. However, we were not able to obtain the solution that roughly corresponds to the part of the curve with the negative gradient in Fig. 5. The solution on this part may be unstable. The profiles of the tangential velocity $v_{\theta}$ of the

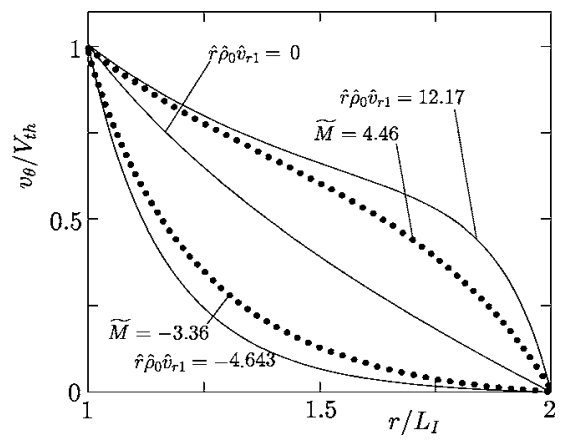

FIG. 9. Profiles of the tangential velocity $v_{\theta}$ at $\mathrm{Kn}=0.005$ (DSMC result for hard-sphere molecules) corresponding to $\left(V_{\mathrm{I}} / V_{\mathrm{th}}, \tilde{M}\right)=(1.0053,-3.36)$ and $(1.0053,4.46)$ in the case of $n_{\mathrm{av}}^{B} / n_{\mathrm{I}}=0.05, n_{\mathrm{II}} / n_{\mathrm{I}}=1.5, \mathrm{~m}^{B} / \mathrm{m}^{A}=1$, and $d^{B} / d^{A}=1\left(L_{\mathrm{II}} / L_{\mathrm{I}}=2, T_{\mathrm{II}} / T_{\mathrm{I}}=1\right.$, and $\left.V_{\mathrm{II}}=0\right)$. The corresponding results in the continuum limit, i.e., the profiles at $\left(V_{\mathrm{I}} / V_{\mathrm{th}}, \hat{r} \hat{\rho}_{0} \hat{v}_{r 1}\right)=(1.0053,-4.643)$, $(1.0053,0)$, and $(1.0053,12.17)$, are also shown by the solid line.
DSMC result corresponding to the points $\left(V_{\mathrm{I}} / V_{\mathrm{th}}, \tilde{M}\right)$ $=(1.0053,-3.36)$ and $(1.0053,4.46)$ for $n_{\mathrm{av}}^{B} / n_{\mathrm{I}}=0.05$ are shown in Fig. 9 together with the corresponding result in the continuum limit, i.e., $\quad\left(V_{\mathrm{I}} / V_{\mathrm{th}}, \hat{r} \hat{\rho}_{0} \hat{v}_{r 1}\right)=(1.0053,-4.643)$, $(1.0053,0)$, and $(1.0053,12.17)$.

In Figs. 8 and 9, the DSMC results at $\mathrm{Kn}=0.005$ still deviate significantly from the corresponding continuum limit. The discrepancy should reduce if we are able to carry out the DSMC computation for smaller Knudsen numbers. However, for the reason described in the last paragraph in Sec. IV B, such a computation is extremely hard. The computational system used for Figs. 8 and 9 is the same as that for $\mathrm{Kn}=0.005$ in Fig. 3 (see the last paragraph in Sec. IV B).

\section{ACKNOWLEDGMENTS}

The authors thank Professor Shigeru Takata for his valuable discussions. This work is supported by the Grants-inAid for Scientific Research (Nos. 17360041 and 16001161) from JSPS and by the Center of Excellence for Research and Education on Complex Functional Mechanical Systems. The junior author (H.Y.) participated in the present work as a JSPS Research Fellow.

\section{APPENDIX A: MACROSCOPIC QUANTITIES OF THE HILBERT EXPANSION}

The explicit expressions of the macroscopic quantities of the Hilbert expansion by $\hat{F}_{m}^{\alpha}$ are summarized. The coefficients of each gas $h_{m}^{\alpha}$ are

$$
\begin{aligned}
& \hat{n}_{0}^{\alpha}=\int \hat{F}_{0}^{\alpha} \mathrm{d} \zeta, \\
& \hat{v}_{r 0}^{\alpha}=\frac{1}{\hat{n}_{0}^{\alpha}} \int \zeta_{r} \hat{F}_{0}^{\alpha} \mathrm{d} \zeta, \\
& \hat{v}_{\theta 0}^{\alpha}=\frac{1}{\hat{n}_{0}^{\alpha}} \int \zeta_{\theta} \hat{F}_{0}^{\alpha} \mathrm{d} \zeta, \\
& \hat{p}_{0}^{\alpha}=\hat{n}_{0}^{\alpha} \hat{T}_{0}^{\alpha}=\frac{2}{3} \int\left[\left(\zeta_{r}-\hat{v}_{r 0}^{\alpha}\right)^{2}+\left(\zeta_{\theta}-\hat{v}_{\theta 0}^{\alpha}\right)^{2}+\zeta_{z}^{2}\right] \\
& \quad \times \hat{m}^{\alpha} \hat{F}_{0}^{\alpha} \mathrm{d} \zeta,
\end{aligned}
$$

and

$$
\begin{aligned}
& \hat{n}_{1}^{\alpha}=\int \hat{F}_{1}^{\alpha} \mathrm{d} \zeta, \\
& \hat{v}_{r 1}^{\alpha}=\frac{1}{\hat{n}_{0}^{\alpha}} \int \zeta_{r} \hat{F}_{1}^{\alpha} \mathrm{d} \zeta-\frac{\hat{n}_{1}^{\alpha}}{\hat{n}_{0}^{\alpha}} \hat{v}_{r 0}^{\alpha}, \\
& \hat{v}_{\theta 1}^{\alpha}=\frac{1}{\hat{n}_{0}^{\alpha}} \int \zeta_{\theta} \hat{F}_{1}^{\alpha} \mathrm{d} \zeta-\frac{\hat{n}_{1}^{\alpha}}{\hat{n}_{0}^{\alpha}} \hat{v}_{\theta 0}^{\alpha},
\end{aligned}
$$




$$
\begin{aligned}
\hat{p}_{1}^{\alpha}=\hat{n}_{0}^{\alpha} \hat{T}_{1}^{\alpha}+\hat{n}_{1}^{\alpha} \hat{T}_{0}^{\alpha}= & \frac{2}{3} \int\left[\left(\zeta_{r}-\hat{v}_{r 0}^{\alpha}\right)^{2}+\left(\zeta_{\theta}-\hat{v}_{\theta 0}^{\alpha}\right)^{2}+\zeta_{z}^{2}\right] \\
& \times \hat{m}^{\alpha} \hat{F}_{1}^{\alpha} \mathrm{d} \zeta .
\end{aligned}
$$

The coefficients of the total mixture $h_{m}$ are expressed in terms of those of each gas:

$$
\begin{aligned}
& \hat{n}_{0}=\hat{n}_{0}^{A}+\hat{n}_{0}^{B}, \\
& \hat{\rho}_{0}=\hat{m}^{A} \hat{n}_{0}^{A}+\hat{m}^{B} \hat{n}_{0}^{B}, \\
& \hat{v}_{r 0}=\frac{1}{\hat{\rho}_{0}}\left(\hat{m}^{A} \hat{n}_{0}^{A} \hat{v}_{r 0}^{A}+\hat{m}^{B} \hat{n}_{0}^{B} \hat{v}_{r 0}^{B}\right), \\
& \hat{v}_{\theta 0}=\frac{1}{\hat{\rho}_{0}}\left(\hat{m}^{A} \hat{n}_{0}^{A} \hat{v}_{\theta 0}^{A}+\hat{m}^{B} \hat{n}_{0}^{B} \hat{v}_{\theta 0}^{B}\right), \\
& \hat{p}_{0}=\hat{n}_{0} \hat{T}_{0}=\sum_{\alpha=A, B}\left[\hat{p}_{0}^{\alpha}+\frac{2}{3} \hat{m}^{\alpha} \hat{n}_{0}^{\alpha}\left(V_{r 0}^{\alpha}\right)^{2}+\frac{2}{3} \hat{m}^{\alpha} \hat{n}_{0}^{\alpha}\left(V_{\theta 0}^{\alpha}\right)^{2}\right],
\end{aligned}
$$

and

$$
\begin{aligned}
& \hat{n}_{1}=\hat{n}_{1}^{A}+\hat{n}_{1}^{B}, \\
& \hat{\rho}_{1}=\hat{m}^{A} \hat{n}_{1}^{A}+\hat{m}^{B} \hat{n}_{1}^{B}, \\
& \hat{v}_{r 1}=\frac{1}{\hat{\rho}_{0}}\left[\sum_{\alpha=A, B}\left(\hat{m}^{\alpha} \hat{n}_{0}^{\alpha} \hat{v}_{r 1}^{\alpha}+\hat{m}^{\alpha} \hat{n}_{1}^{\alpha} \hat{v}_{r 0}^{\alpha}\right)-\hat{\rho}_{1} \hat{v}_{r 0}\right], \\
& \hat{v}_{\theta 1}=\frac{1}{\hat{\rho}_{0}}\left[\sum_{\alpha=A, B}\left(\hat{m}^{\alpha} \hat{n}_{0}^{\alpha} \hat{v}_{\theta 1}^{\alpha}+\hat{m}^{\alpha} \hat{n}_{1}^{\alpha} \hat{v}_{\theta 0}^{\alpha}\right)-\hat{\rho}_{1} \hat{v}_{\theta 0}\right] \\
& \hat{p}_{1}=\hat{n}_{0} \hat{T}_{1}+\hat{n}_{1} \hat{T}_{0}=\sum_{\alpha=A, B}\left\{\hat{p}_{1}^{\alpha}+\frac{2}{3} \hat{m}^{\alpha} \hat{n}_{1}^{\alpha}\left[\left(V_{r 0}^{\alpha}\right)^{2}+\left(V_{\theta 0}^{\alpha}\right)^{2}\right]\right.
\end{aligned}
$$

where

$$
V_{r m}^{\alpha}=\hat{v}_{r m}^{\alpha}-\hat{v}_{r m}, \quad V_{\theta m}^{\alpha}=\hat{v}_{\theta m}^{\alpha}-\hat{v}_{\theta m} .
$$

\section{APPENDIX B: HILBERT SOLUTION $\hat{\boldsymbol{F}}_{1}^{\alpha}$}

In this appendix the index $\gamma$ is used to represent the labels $A$ and $B$ of the gas species, in addition to $\alpha$ and $\beta$. The first-order Hilbert solution $\hat{F}_{1}^{\alpha}$ is given in the following form:

$$
\begin{aligned}
\hat{F}_{1}^{\alpha}= & \hat{F}_{0}^{\alpha}\left[\frac{\hat{p}_{1}^{\alpha}}{\hat{p}_{0}^{\alpha}}+2 \hat{m}^{\alpha} \frac{\hat{v}_{r 1} \widetilde{\zeta}_{r}+\hat{v}_{\theta 1} \widetilde{\zeta}_{\theta}}{\hat{T}_{0}^{1 / 2}}+\frac{\hat{T}_{1}}{\hat{T}_{0}}\left(\hat{m}^{\alpha} \widetilde{\zeta}^{2}-\frac{5}{2}\right)\right. \\
& -\widetilde{\zeta}_{r} A^{\alpha}(\widetilde{\zeta}) \frac{1}{\hat{p}_{0}} \frac{\mathrm{d} \hat{T}_{0}}{\mathrm{~d} \hat{r}}-\widetilde{\zeta}_{r} \widetilde{\zeta}_{\theta} B^{\alpha}(\widetilde{\zeta}) \frac{\hat{T}_{0}^{1 / 2}}{\hat{p}_{0}}\left(\frac{\mathrm{d} \hat{v}_{\theta 0}}{\mathrm{~d} \hat{r}}-\frac{\hat{v}_{\theta 0}}{\hat{r}}\right) \\
& \left.-\tilde{\zeta}_{r} \sum_{\beta=A, B} D^{(\beta) \alpha}(\widetilde{\zeta}) \frac{1}{\hat{n}_{0} \hat{p}_{0}}\left(\frac{\mathrm{d} \hat{p}_{0}^{\beta}}{\mathrm{d} \hat{r}}-\frac{2 \hat{m}^{\beta} \hat{n}_{0}^{\beta} \hat{v}_{\theta 0}^{2}}{\hat{r}}\right)\right],
\end{aligned}
$$

where

$$
\begin{aligned}
& \tilde{\zeta}_{r}=\frac{\zeta_{r}}{\hat{T}_{0}^{1 / 2}}, \quad \tilde{\zeta}_{\theta}=\frac{\zeta_{\theta}-\hat{v}_{\theta 0}}{\hat{T}_{0}^{1 / 2}}, \quad \tilde{\zeta}_{z}=\frac{\zeta_{z}}{\hat{T}_{0}^{1 / 2}}, \\
& \tilde{\zeta}^{2}=\widetilde{\zeta}_{r}^{2}+\widetilde{\zeta}_{\theta}^{2}+\widetilde{\zeta}_{z}^{2} .
\end{aligned}
$$

The functions $A^{\alpha}(\zeta), B^{\alpha}(\zeta)$, and $D^{(\beta) \alpha}(\zeta)$ are the solutions of the following integral equations: $:^{28,50}$

$$
\begin{aligned}
& \sum_{\beta=A, B} K^{\beta \alpha} \chi_{0}^{\beta} \widetilde{L}_{T}^{\beta \alpha}\left(\zeta_{r} A^{\beta}, \zeta_{r} A^{\alpha}\right)=-\zeta_{r}\left(\hat{m}^{\alpha} \zeta^{2}-\frac{5}{2}\right), \\
& \text { subsidiary condition: } \sum_{\alpha=A, B} \hat{m}^{\alpha} \chi_{0}^{\alpha} I_{4}^{\alpha}\left(A^{\alpha}\right)=0,
\end{aligned}
$$

$$
\sum_{\beta=A, B} K^{\beta \alpha} \chi_{0}^{\beta} \widetilde{L}_{T}^{\beta \alpha}\left(\zeta_{r} \zeta_{\theta} B^{\beta}, \zeta_{r} \zeta_{\theta} B^{\alpha}\right)=-2 \hat{m}^{\alpha} \zeta_{r} \zeta_{\theta}
$$

$$
\begin{aligned}
& \sum_{\beta=A, B} K^{\beta \alpha} \chi_{0}^{\alpha} \chi_{0}^{\beta} \widetilde{L}_{T}^{\beta \alpha}\left(\zeta_{r} D^{(\gamma) \beta}, \zeta_{r} D^{(\gamma) \alpha}\right)=-\zeta_{r}\left(\delta_{\alpha \gamma}-\frac{\hat{m}^{\alpha} n_{0}^{\alpha}}{\hat{\rho}_{0}}\right), \\
& \text { subsidiary condition: } \sum_{\alpha=A, B} \hat{m}^{\alpha} \chi_{0}^{\alpha} I_{4}^{\alpha}\left(D^{(\beta) \alpha}\right)=0,
\end{aligned}
$$

where $\zeta=\left(\zeta_{r}^{2}+\zeta_{\theta}^{2}+\zeta_{z}^{2}\right)^{1 / 2}$. The operators $\tilde{L}_{T}^{\beta \alpha}(f, g)$ and $I_{n}^{\alpha}(F)$ are defined as follows:

$$
\begin{aligned}
& \widetilde{L}_{T}^{\beta \alpha}(f, g)= \int\left[f\left(\boldsymbol{\zeta}_{*}^{\prime}\right)+g\left(\boldsymbol{\zeta}^{\prime}\right)-f\left(\boldsymbol{\zeta}_{*}\right)-g(\boldsymbol{\zeta})\right] \\
& \times E^{\beta}\left(\zeta_{*}\right) \hat{B}_{T}^{\beta \alpha}(|\mathbf{e} \cdot \hat{\boldsymbol{V}}| / \hat{V}, \hat{V}) \mathrm{d} \Omega(\mathbf{e}) \mathrm{d} \boldsymbol{\zeta}_{*}, \\
& \hat{B}_{T}^{\beta \alpha}(|\mathbf{e} \cdot \hat{\boldsymbol{V}}| / \hat{V}, \hat{V})=\hat{B}^{\beta \alpha}\left(|\mathbf{e} \cdot \hat{\boldsymbol{V}}| / \hat{V}, \hat{V} \hat{T}_{0}^{1 / 2}\right) / \hat{T}_{0}^{1 / 2}, \\
& I_{n}^{\alpha}(F)=\frac{8 \pi}{15}\left(\frac{\hat{m}^{\alpha}}{\pi}\right)^{3 / 2} \int_{0}^{\infty} \zeta^{n} F(\zeta) \exp \left(-\hat{m}^{\alpha} \zeta^{2}\right) \mathrm{d} \zeta,
\end{aligned}
$$

where

$$
\begin{aligned}
& E^{\beta}\left(\zeta_{*}\right)=\left(\frac{\hat{m}^{\beta}}{\pi}\right)^{3 / 2} \exp \left(-\hat{m}^{\beta} \zeta_{*}^{2}\right), \\
& \zeta_{*}=\left(\zeta_{*_{r}}^{2}+\zeta_{* \theta}^{2}+\zeta_{*_{z}}^{2}\right)^{1 / 2} .
\end{aligned}
$$

The transport coefficients $\hat{\mu}, \hat{\lambda}, \hat{D}_{A B}$, and $k_{T}$ in Eqs. (58)-(60) are defined by means of the functions $A^{\alpha}, B^{\alpha}$, and $D^{(\beta) \alpha}$ as follows: $:^{28,50}$

$$
\hat{D}_{A B}=\chi_{0}^{A} \chi_{0}^{B}\left(\hat{\Delta}_{A A}+\hat{\Delta}_{B B}-\hat{\Delta}_{A B}-\hat{\Delta}_{B A}\right),
$$




$$
\begin{aligned}
& \hat{D}_{T}=\chi_{0}^{A} \chi_{0}^{B}\left(\hat{D}_{T A}-\hat{D}_{T B}\right), \\
& \hat{\mu}=\sum_{\alpha=A, B} \chi_{0}^{\alpha} \hat{\mu}^{\alpha}, \quad \hat{\lambda}^{\prime}=\sum_{\alpha=A, B} \chi_{0}^{\alpha} \hat{\lambda}^{\prime \alpha}, \\
& k_{T}=\frac{\hat{D}_{T}}{\hat{D}_{A B}}, \quad \hat{\lambda}=\hat{\lambda}^{\prime}-k_{T} \frac{\hat{D}_{T}}{\chi_{0}^{A} \chi_{0}^{B}},
\end{aligned}
$$

where

$$
\begin{aligned}
& \hat{\Delta}_{\alpha \beta}=\frac{5}{2} I_{4}^{\alpha}\left(D^{(\beta) \alpha}\right), \\
& \hat{D}_{T \alpha}=\frac{5}{2} I_{4}^{\alpha}\left(A^{\alpha}\right), \\
& \hat{\mu}^{\alpha}=\hat{m}^{\alpha} I_{6}^{\alpha}\left(B^{\alpha}\right), \\
& \hat{\lambda}^{\prime \alpha}=\frac{5}{2} I_{4}^{\alpha}\left(\left[\hat{m}^{\alpha} \zeta^{2}-\frac{5}{2}\right] A^{\alpha}\right) .
\end{aligned}
$$

From the subsidiary conditions and appropriate integration of the integral equations in Eqs. (B3) and (B5), the following relations are derived, with the help of which the form of Eqs. (58)-(60) is obtained:

$$
\begin{aligned}
& \hat{\Delta}_{\alpha \beta}=\hat{\Delta}_{\beta \alpha}, \\
& \sum_{\beta=A, B} \hat{m}^{\beta} \chi_{0}^{\beta} \hat{\Delta}_{\alpha \beta}=0, \\
& \sum_{\beta=A, B} \hat{m}^{\beta} \chi_{0}^{\beta} \hat{D}_{T \beta}=0 .
\end{aligned}
$$

For hard-sphere molecules, the coefficients $\hat{\mu}, \hat{\lambda}, \hat{D}_{A B}$, and $k_{T}$ are independent of the temperature $\hat{T}_{0}$. A numerical database for these coefficients in this case as functions of $\chi_{0}^{A}$ has been constructed in Ref. 47, and it has been used in obtaining the numerical solution presented in Sec. IV.

${ }^{1}$ C. Cercignani and F. Sernagiotto, "Cylindrical Couette flow of a rarefied gas," Phys. Fluids 10, 1200 (1967).

${ }^{2} \mathrm{~K}$. Nanbu, "Analysis of cylindrical Couette flows by use of the direction simulation method," Phys. Fluids 27, 2632 (1984).

${ }^{3}$ R. E. Khayat and B. C. Eu, "Nonlinear transport processes and fluid dynamics: Cylindrical Couette flow of Lennard-Jones fluids," Phys. Rev. A 38, 2492 (1988); erratum, ibid. 40, 1150 (1989).

${ }^{4}$ R. E. Khayat and B. C. Eu, "Generalized hydrodynamics, normal-stress effects, and velocity slips in the cylindrical Couette flow of Lennard-Jones fluids," Phys. Rev. A 39, 728 (1989); erratum, ibid. 40, 1150 (1989).

${ }^{5}$ F. Sharipov and G. Kremer, "Linear Couette flow between two rotating cylinders,” Eur. J. Mech. B/Fluids 15, 493 (1996).

${ }^{6}$ F. Sharipov and G. Kremer, "Nonlinear Couette flow between two rotating cylinders," Transp. Theory Stat. Phys. 25, 217 (1996).

${ }^{7}$ K. W. Tibbs, F. Baras, and A. L. Garcia, "Anomalous flow profile due to the curvature effect on slip length,” Phys. Rev. E 56, 2282 (1997).

${ }^{8}$ L. M. de Socio, N. Ianiro, and L. Marino, "Effects of the centrifugal forces on a gas between rotating cylinders," J. Thermophys. Heat Transfer 14, 269 (2000)

${ }^{9}$ L. M. de Socio and L. Marino, "Simulation and modelling of flows between rotating cylinders. Influence of Knudsen number," Math. Models Meth. Appl. Sci. 10, 73 (2000).

${ }^{10}$ K. Aoki, H. Yoshida, T. Nakanishi, and A. L. Garcia, "Inverted velocity profile in the cylindrical Couette flow of a rarefied gas," Phys. Rev. E 68, 016302 (2003).

${ }^{11} \mathrm{~S}$. Yuhong, R. W. Barber, and D. R. Emerson, "Inverted velocity profiles in rarefied cylindrical Couette gas flow and the impact of the accommodation coefficient," Phys. Fluids 17, 047102 (2005).

${ }^{12}$ Y. Sone, H. Sugimoto, and K. Aoki, "Cylindrical Couette flows of a rarefied gas with evaporation and condensation: Reversal and bifurcation of flows," Phys. Fluids 11, 476 (1999).

${ }^{13}$ Y. Sone, T. Ohwada, and Y. Makihara, "Bifurcation in cylindrical Couette flow with evaporation and condensation: Effect of initial condition," in Rarefied Gas Dynamics, edited by R. Brun, R. Campargue, R. Gatignol, and J.-C. Lengrand (Cépaduès-Éditions, Toulouse, 1999), Vol. 1, p. 511.

${ }^{14}$ Y. Sone and T. Doi, "Analytical study of bifurcation of a flow of a gas between coaxial circular cylinders with evaporation and condensation," Phys. Fluids 12, 2639 (2000).

${ }^{15}$ Y. Sone, M. Handa, and H. Sugimoto, "Bifurcation studies of flows of a gas between rotating coaxial circular cylinders with evaporation and condensation by the Boltzmann system," Transp. Theory Stat. Phys. 31, 299 (2002).

${ }^{16}$ L. Arkeryd and A. Nouri, "The stationary nonlinear Boltzmann equation in a Couette setting with multiple, isolated $L^{q}$-solutions and hydrodynamic limits," J. Stat. Phys. 118, 849 (2005).

${ }^{17} \mathrm{~K}$. Aoki and Y. Sone, "Gas flows around the condensed phase with strong evaporation or condensation: Fluid dynamic equation and its boundary condition on the interface and their application," in Advances in Kinetic Theory and Continuum Mechanics, edited by R. Gatignol and Soubbaramayer (Springer, Berlin, 1991), p. 43.

${ }^{18}$ Y. Sone, S. Takata, and H. Sugimoto, "The behavior of a gas in the continuum limit in the light of kinetic theory: The case of cylindrical Couette flows with evaporation and condensation," Phys. Fluids 8, 3403 (1996); erratum, ibid. 10, 1239 (1998).

${ }^{19}$ Y. Sone, K. Aoki, S. Takata, H. Sugimoto, and A. V. Bobylev, "Inappropriateness of the heat-conduction equation for description of a temperature field of a stationary gas in the continuum limit: Examination by asymptotic analysis and numerical computation of the Boltzmann equation," Phys. Fluids 8, 628 (1996); erratum, ibid. 8, 841 (1996).

${ }^{20}$ Y. Sone, "Continuum gas dynamics in the light of kinetic theory and new features of rarefied gas flows," in Rarefied Gas Dynamics, edited by C. Shen (Peking University Press, Beijing, 1997), p. 3.

${ }^{21}$ Y. Sone, "Flows induced by temperature fields in a rarefied gas and their ghost effect on the behavior of a gas in the continuum limit," Annu. Rev. Fluid Mech. 32, 779 (2000).

${ }^{22}$ Y. Sone, Kinetic Theory and Fluid Dynamics (Birkhäuser, Boston, 2002).

${ }^{23}$ Y. Sone, M. Handa, and T. Doi, "Ghost effect and bifurcation in a gas between coaxial circular cylinders with different temperatures," Phys. Fluids 15, 2903 (2003).

${ }^{24}$ Y. Sone and T. Doi, "Ghost effect of infinitesimal curvature in the plane Couette flow of a gas in the continuum limit," Phys. Fluids 16, 952 (2004).

${ }^{25}$ Y. Sone, Molecular Gas Dynamics: Theory, Techniques, and Applications (Birkhäuser, Boston, 2006).

${ }^{26} \mathrm{~S}$. Takata and K. Aoki, "Two-surface problems of a multicomponent mixture of vapors and noncondensable gases in the continuum limit in the light of kinetic theory," Phys. Fluids 11, 2743 (1999).

${ }^{27}$ M. N. Kogan, Rarefied Gas Dynamics (Plenum, New York, 1969).

${ }^{28} \mathrm{~S}$. Chapman and T. G. Cowling, The Mathematical Theory of NonUniform Gases, 3rd ed. (Cambridge University Press, Cambridge, 1995).

${ }^{29}$ C. Cercignani, Rarefied Gas Dynamics: From Basic Concepts to Actual Calculations (Cambridge University Press, 2000).

${ }^{30}$ F. Reif, Fundamentals of Statistical and Thermal Physics (McGraw-Hill, New York, 1965).

${ }^{31}$ C. Cercignani and M. Lampis, "Kinetic models for gas-surface interactions," Transp. Theory Stat. Phys. 1, 101 (1971).

${ }^{32}$ G. Nagayama and T. Tsuruta, "A general expression for the condensation coefficient based on transition state theory and molecular dynamics simulation," J. Chem. Phys. 118, 1392 (2003).

${ }^{33}$ R. Meland, A. Frezzotti, T. Ytrehus, and B. Hafskjold, "Nonequilibrium molecular-dynamics simulation of net evaporation and net condensation, and evaluation of the gas-kinetic boundary condition at the interphase," Phys. Fluids 16, 223 (2004).

${ }^{34}$ T. Ishiyama, T. Yano, and S. Fujikawa, "Molecular dynamics study of kinetic boundary condition at an interface between argon vapor and its condensed phase," Phys. Fluids 16, 2899 (2004).

${ }^{35}$ A. Frezzotti, L. Gibelli, and S. Lorenzani, "Mean field kinetic theory de- 
scription of evaporation of a fluid into vacuum," Phys. Fluids 17, 012102 (2005).

${ }^{36}$ T. Ishiyama, T. Yano, and S. Fujikawa, "Kinetic boundary condition at a vapor-liquid interface," Phys. Rev. Lett. 95, 084504 (2005).

${ }^{37}$ Y. Sone, "Asymptotic theory of flow of rarefied gas over a smooth boundary I," in Rarefied Gas Dynamics, edited by L. Trilling and H. Y. Wachman (Academic, New York, 1969), p. 243.

${ }^{38}$ Y. Sone, "Asymptotic theory of flow of rarefied gas over a smooth boundary II," in Rarefied Gas Dynamics, edited by D. Dini (Editrice Tecnico Scientifica, Pisa, Italy, 1971), Vol. II, p. 737.

${ }^{39}$ Y. Sone, "Asymptotic theory of a steady flow of a rarefied gas past bodies for small Knudsen numbers," in Advances in Kinetic Theory and Continuum Mechanics, edited by R. Gatignol and Soubbaramayer (Springer, Berlin, 1991), p. 19.

${ }^{40}$ K. Aoki, S. Takata, and S. Taguchi, "Vapor flows with evaporation and condensation in the continuum limit: Effect of a trace of noncondensable gas," Eur. J. Mech. B/Fluids 22, 51 (2003).

${ }^{41}$ K. Aoki, S. Takata, and S. Kosuge, "Vapor flows caused by evaporation and condensation on two parallel plane surfaces: Effect of the presence of a noncondensable gas," Phys. Fluids 10, 1519 (1998).

${ }^{42} \mathrm{~S}$. Taguchi, K. Aoki, and S. Takata, "Vapor flows in the continuum limit in the presence of a small amount of noncondensable gas," Phys. Fluids 16, 4105 (2004).
${ }^{43}$ A. V. Bobylev, "Quasistationary hydrodynamics for the Boltzmann equation," J. Stat. Phys. 80, 1063 (1995).

${ }^{44}$ S. Takata, K. Aoki, and T. Muraki, "Behavior of a vapor-gas mixture between two parallel plane condensed phases in the continuum limit," in Rarefied Gas Dynamics, edited by R. Brun, R. Campargue, R. Gatignol, and J.-C. Lengrand (Cépaduès-Éditions, Toulouse, 1999), Vol. 1, p. 479.

${ }^{45}$ S. Takata and K. Aoki, "The ghost effect in the continuum limit for a vapor-gas mixture around condensed phases: Asymptotic analysis of the Boltzmann equation," Transp. Theory Stat. Phys. 30, 205 (2001); erratum, ibid. 31, 289 (2002).

${ }^{46} \mathrm{~S}$. Takata, "Kinetic theory analysis of the two-surface problem of a vaporvapor mixture in the continuum limit," Phys. Fluids 16, 2182 (2004).

${ }^{47}$ S. Takata, S. Yasuda, K. Aoki, and T. Shibata, "Various transport coefficients occurring in binary gas mixtures and their database," in Rarefied Gas Dynamics, edited by A. D. Ketsdever and E. P. Muntz (AIP, Melville, NY, 2003), p. 106.

${ }^{48}$ G. A. Bird, Molecular Gas Dynamics (Oxford University Press, Oxford, 1976).

${ }^{49}$ G. A. Bird, Molecular Gas Dynamics and the Direct Simulation of Gas Flows (Oxford University Press, Oxford, 1994).

${ }^{50} \mathrm{C}$. F. Delale, "The Hilbert theory of transport phenomena in multiple gas mixtures," J. Chem. Phys. 83, 3069 (1985). 